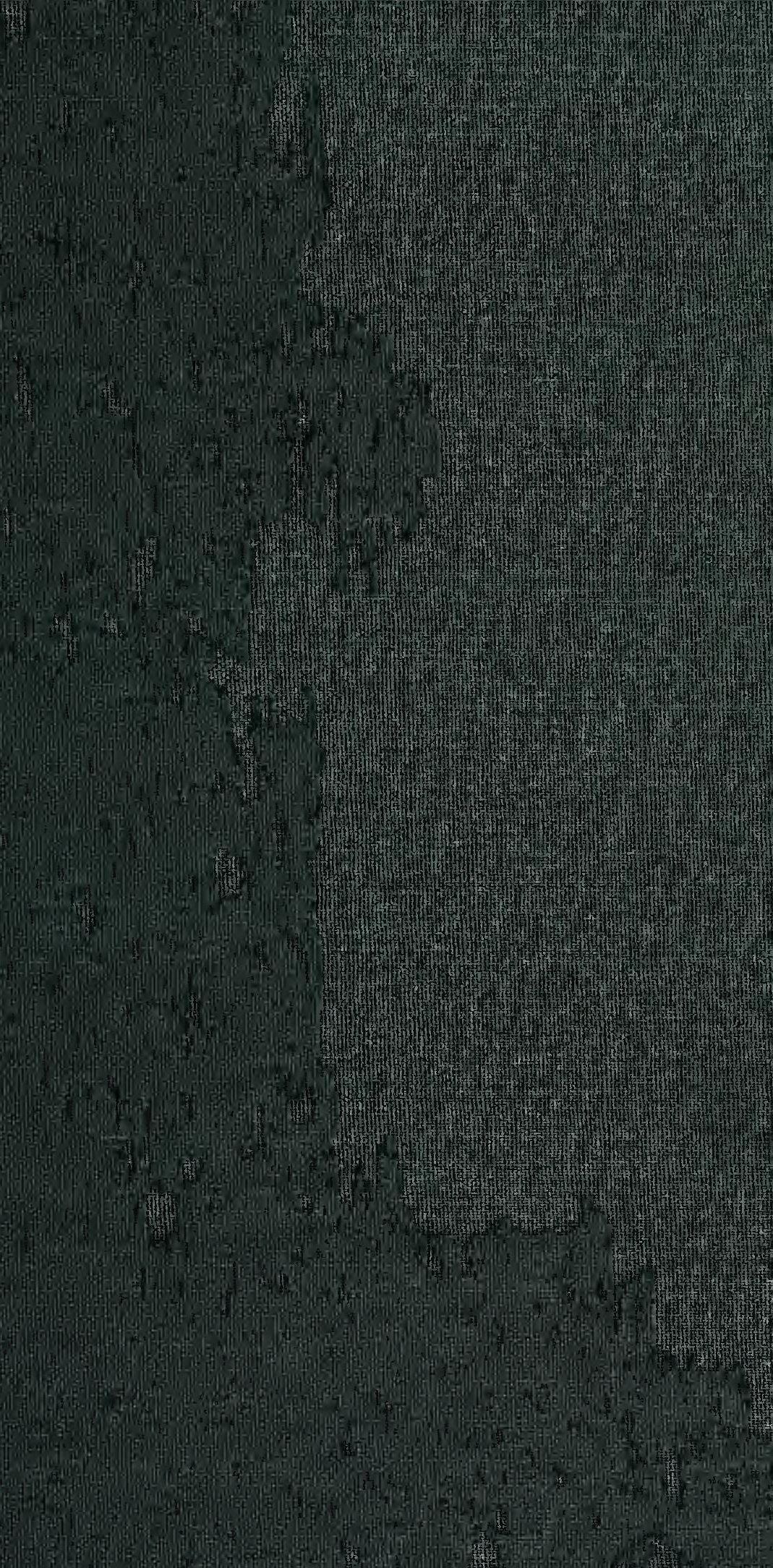




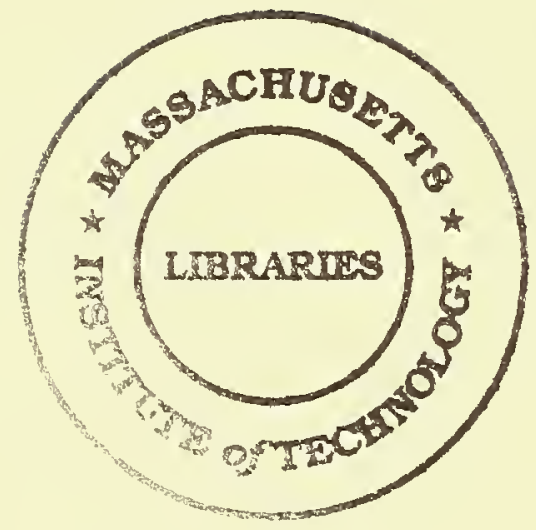


Digitized by the Internet Archive in 2011 with funding from

Boston Library Consortium Member Libraries

http://www.archive.org/details/optimalcollusion00athe 



\section{working paper department of economics}

\section{Optimal Collusion With Private Information}

Susan Athey

Kyle Bagwell

No. $99-17$

October 1999

\section{massachusetts institute of technology}

50 memorial drive cambridge, mass. 02139 


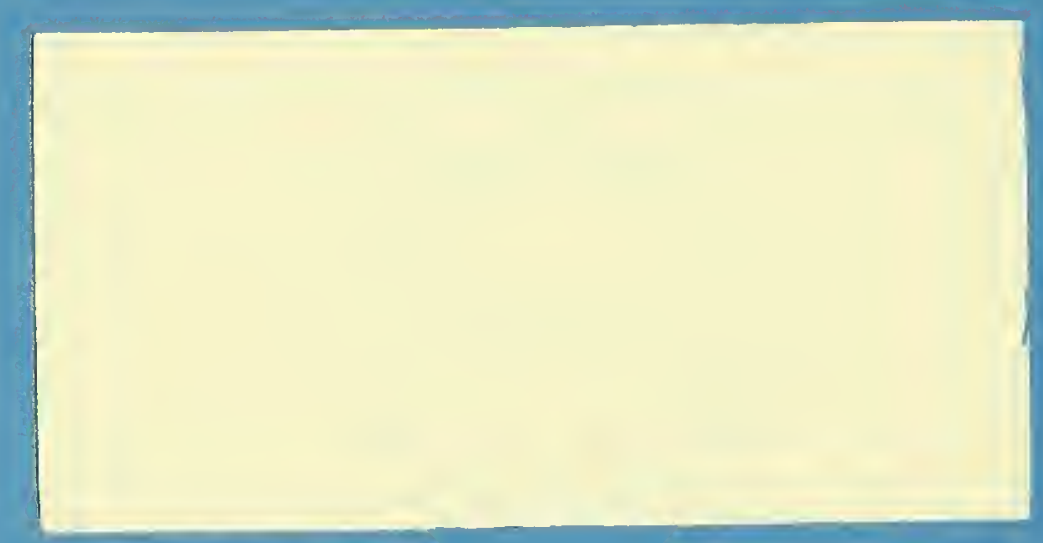




\section{WORKING PAPER \\ DEPARTMENT \\ OF ECONOMICS}

Optimal Collusion With Private Information

Susan Athey

Kyle Bagwell

No. $99-17$

October 1999

\section{MASSACHUSETTS \\ INSTITUTE OF TECHNOLOGY}

50 MEMORIAL DRIVE

CAMBRIDGE, MASS. 02142 

We consider an infinitely-repeated Bertrand game, in which prices are perfectly observed and each firm receives a privately-observed, i.i.d. cost shock in each period. In our base model, side-payments are prohibited. In order for a cartel to achieve productive efficiency, firms that receive a high cost draw in the current period must be induced to give up market share. To provide this incentive, firms may need to reduce current prices, make compensating adjustments in future market shares or allow for future price wars. In the most profitable collusive schemes, firms implement productive efficiency, but they do not resort to low prices or future price wars; instead, firms with bad cost draws may be favored with higher expected market share in future periods. If types are discrete, there exists a discount factor strictly less than one above which first-best profits can be attained purely through history-dependent reallocation of market share between equally-efficient firms. We provide further characterizations of equilibrium play as well as several computational examples.

We next consider the role of three characteristics of the institutional environment. First, we examine the costs and benefits to the cartel of explicit communication about cost types in a given period. We show that firms may find it beneficial to communicate after some histories but not others. Second, we show that if firm identities cannot be tracked over time (so that firm-specific future market-share favors are unavailable), the best collusive scheme sacrifices all productive efficiency. Third, we examine the role of explicit sidepayments, which may entail inefficiencies if they are illegal and bear the risk of detection. Unless side-payments are perfectly efficient, optimal collusive equilibria are non-stationary and thus involve the use of future market-share favors. 


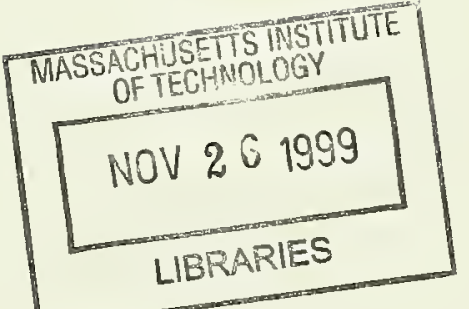




\title{
Optimal Collusion with Private Information
}

\author{
Susan Athey and Kyle Bagwell \\ First Draft: May, 1998. This Draft: September, 1999.
}

ABSTRACT: We consider an infinitely-repeated Bertrand game, in which prices are perfectly observed and each firm receives a privately-observed, i.i.d. cost shock in each period. In our base model, side-payments are prohibited. In order for a cartel to achieve productive efficiency, firms that receive a high cost draw in the current period must be induced to give up market share. To provide this incentive, firms may need to reduce current prices, make compensating adjustments in future market shares or allow for future price wars. In the most profitable collusive schemes, firms implement productive efficiency, but they do not resort to low prices or future price wars; instead, firms with bad cost draws may be favored with higher expected market share in future periods. If types are discrete, there exists a discount factor strictly less than one above which first-best profits can be attained purely through history-dependent reallocation of market share between equally-efficient firms. We provide further characterizations of equilibrium play as well as several computational examples.

We next consider the role of three characteristics of the institutional environment. First, we examine the costs and benefits to the cartel of explicit communication about cost types in a given period. We show that firms may find it beneficial to communicate after some histories but not others. Second, we show that if firm identities cannot be tracked over time (so that firm-specific future market-share favors are unavailable), the best collusive scheme sacrifices all productive efficiency. Third, we examine the role of explicit side-payments, which may entail inefficiencies if they are illegal and bear the risk of detection. Unless side-payments are perfectly efficient, optimal collusive equilibria are non-stationary and thus involve the use of future market-share favors.

JEL Classification Numbers: C73, L13, L41.

Keywords: Collusion, cartel design, repeated games, private information, mechanism design, auctions, information-sharing, communication.

${ }^{*}$ M.I.T. and NBER (Athey) and Columbia University and NBER (Bagwell). We are especially grateful to Pavel Grigoriev for exceptional research assistance, and to Eric Maskin for providing detailed comments on an earlier draft. We would like to thank Glenn Ellison, Wally Mullin, David Pearce, Lars Stole, Jean Tirole and seminar participants at Barcelona (Pompeu Fabra), Berkeley, Federal Reserve Bank Board of Governors, Harvard, M.I.T., Minneapolis Federal Reserve Bank, S.I.T.E., Stanford, Toulouse (I.D.E.I.), UC-San Diego, the Summer Conference on I.O. in Toronto, and the Stony Brook Game Theory Conference for helpful discussions. We thank the NSF (Athey: SBR-9631760; Bagwell: SES-9905460) for generous financial support. Athey thanks the Cowles Foundation at Yale University for hospitality in 1997-1998, when much of this research was conducted. 



\section{Introduction}

In this paper, we consider an infinitely-repeated Bertrand game, in which each firm is privately informed of its unit cost level in each period, and the unit-cost realization is i.i.d. across firms and time. We thus represent collusion in the context of a repeated hidden-information model with publicly-observed actions (prices). ${ }^{1}$ Building on work by Abreu, Pearce and Stacchetti (1990) and Fudenberg, Levine and Maskin (1994), we use the tools of dynamic programming to recast the characterization of optimal Perfect Public Equilibria (PPE) in terms of a static mechanism design problem, in which transfers across firms are drawn from a restricted set (namely, the set of PPE continuation values). Thus, we show how firms who are prohibited from making side-payments can still implement a self-enforcing scheme where, in place of an explicit monetary transfer from one firm to another, the collusive mechanism specifies that one firm is favored over another in future play.

Our analysis is motivated by the question of how collusive conduct varies across different market and legal environments. In particular, the design and enforcement of anti-trust policies vary widely across countries and industries, and the different manifestations of anti-trust policies naturally affect the organization of collusive activity. ${ }^{2}$ If the anti-trust environment is permissive, then firms may set up industry associations in which they confirm the terms of their agreement, communicate about current conditions, keep records of past experiences and perhaps even exchange side-payments. ${ }^{3}$ In less permissive anti-trust environments, firms still may communicate as to current conditions, but the conversations may occur surreptitiously, in "smoke-filled rooms," and explicit monetary transfers may be avoided. Finally, a vigorous anti-trust policy might even deter some forms of communication among firms. This latter possibility is the evident aim of current U.S. policy, which holds that communication among firms as to current prices is a per se violation. ${ }^{4}$

While anti-trust policies clearly influence the organizational structure of the collusive

${ }^{1}$ Our model of hidden information can be contrasted with the hidden-action model of Green and Porter (1984) and Abreu, Pearce and Stacchetti (1986), as well as the model of observable demand fluctuations pioneered by Rotemberg and Saloner (1986).

${ }^{2}$ Levinsohn (1995) describes the significant variation in anti-trust law and enforcement that is found across countries. Significant variation also occurs within countries and across industries; for example, in many countries, the legal stance toward cartels is more tolerant in export industries.

${ }^{3}$ For example, anti-trust policy with respect to collusive conduct is often permissive in Japan, and McMillan (1985) discusses that colluding firms there sometimes form "dangos" within which communication and even side-payments are found. As Stocking and Watkins (1946) detail, U.S. anti-trust policy was also relatively permissive in the first part of the 20th century, and industry associations in which firms shared information and records, and exchanged side-payments, were then common. Sophisticated cartels of this nature were found in the stcel, aluminum, incandescent electric lamp, and sugar (as Genesove and Mullin (1998, 1999) describe) industries.

${ }^{4}$ However, it is not illegal to share production plans; see, for example, Doyle and Snyder's (1998) analysis of information sharing in the motor vehicle industry. 
endeavor, the implications for market conduct are more subtle. For example, how, if at all, does the ability to communicate influence prices, market shares and profits? And how might these variables be affected, if firms were able as well to make direct side-payments to one another (perhaps at some cost, if side-payments are illegal but detection occurs with some intermediate probability)? Practically, a theory that addresses such questions might be useful in two main respects. First, it could provide a lens through which to interpret observed (historic or current) collusive conduct in terms of the surrounding market and legal environment. Second, it might provide a framework with which to better anticipate the possible consequences of a change in anti-trust policies for the conduct of collusive firms. ${ }^{5}$

Motivated by these considerations, we analyze in this paper optimal collusion in a range of possible environments. To understand our findings, it is helpful first to recall the tradeoffs that confront the legalized cartel, which uses explicit monetary transfers (sidepayments) that are enforced by binding contracts. An important consideration for the cartel is that production is allocated efficiently over cartel members. As Roberts (1985), Cramton and Palfrey (1990) and Kihlstrom and Vives (1992) have shown, when firms are privately informed as to their respective costs of production, productive efficiency requires communication and transfers between members. Communication enables firms to establish before production the identity of the lowest-cost firm, while transfers (from this firm to the other cartel members) ensure that firms have the incentive to communicate truthfully.

Outside of a legalized cartel, however, the collusive relationship must be self-enforcing. In addition, the collusive environment may restrict the set of instruments with which firms may effect transfers from one to another. Thus, we characterize optimal self-enforcing collusive conduct among privately-informed firms operating in collusive environments that are distinguished on the basis of restrictions on the instruments available to the firms. In our base model, we make the following assumptions: (i). firms can "communicate" with regard to current cost conditions; (ii). firms can use "market-share favors," which requires that the identities of individual firms can be followed over time, and whereby individual firms are treated asymmetrically as a reward or punishment for past behavior; (iii). firms can not make explicit monetary transfers (use "bribes"). After studying this model in some detail, we then analyze the way in which optimal collusion changes as each of the three restrictions is imposed or removed.

Our characterization of the optimal collusive mechanism in the base model highlights the central role played by market-share favors: the prospect of a future favor can provide a high-cost firm with the incentive to truthfully acknowledge its current state and accept a low market share in the present. We show that when such favors are possible, the scope for

\footnotetext{
${ }^{5}$ This consideration is perhaps of some special significance in the current environment, as new institutions are being developed in the Europcan Union and the former communist countries, and further as countries increasingly raise compctition-policy concerns in their trade-policy negotiations.
} 
self-enforcing collusion is significant. Indeed, if firms are sufficiently patient, truth-telling can be induced as follows: if a firm announces low cost in the present, it forgoes some future market share only in the event of "ties," where both firms are equally efficient. Formally, we compute a critical discount factor (strictly less than one) above which the cartel can achieve first-best profits in every period. In essence, the cartel uses asymmetric future market-share assignments to duplicate the transfer patterns that would be employed by a legalized cartel, without any efficiency loss.

When firms are less patient, however, it may be costly to implement market-share favors. In particular, when the firms attempt to implement a scheme that favors one firm, the disadvantaged firm may not be willing to give up enough market share in the event of ties. When its assigned market share is too low following a particular cost realization (in particular, when both firms realize low cost), the disadvantaged firm may be tempted to undercut the collusive price and thereby capture the entire market (though a price war may subsequently ensue). In order to mitigate this temptation, firms may find it necessary to lower prices, in order to diminish the gain from undercutting the collusive price. Alternatively, the scheme may require the disadvantaged firm to give up some market share in the state in which it is most efficient. Intuitively, under a scheme that calls for efficient production across firms, the disadvantaged firm has no incentive to undercut the collusive price in the state of the world when it is most efficient, since it already anticipates serving the entire market. Thus, it is relatively easy to induce the disadvantaged firm to give up a little market share in that state.

At the start of the game, less patient firms anticipate the costs associated with using future market-share favors to provide incentives for truthful revelation. They then weigh the disadvantages of future market-share favors against the productive efficiency gains from truth-telling in the present. One theme of our analysis is that such firms may give up productive efficiency rather than lowering prices in the present or bearing large future inefficiency.

In addition to our theoretical characterizations, we offer a numerical computation of several examples that illustrates the manner in which collusive conduct unfolds over time. The computation highlights an empirical implication of our model: optimal collusion generates negative correlation over time in the market shares of firms. Later in the paper, referring to our theoretical and computational findings, we discuss further the extent to which the predictions of our model can be linked to real-world case studies and empirical applications.

We next turn to evaluate each of our three assumptions about the collusive environment. We begin with the role of communication (assumption (i)). In our model, communication allows firms to smoothly divide the market on a state-contingent basis. Without communication, firms must allocate market share in a decentralized fashion, thereby limiting the 
range of market-sharing plans available. Thus, we show that it is often profitable for firms to choose plans that require communication. At the same time, we also find that firms of moderate patience may choose to avoid communication in some periods. Intuitively, when firms do not communicate, a given firm does not know its opponent's cost type when it chooses its price. Accordingly, if the opponent's price varies with cost, then the firm also does not know the exact price that its opponent will choose. Uncertainty about the opponent's price can, in turn, diminish the incentive that the firm has to "cheat" and select a slightly lower price. Therefore, while communication can generate productive efficiency gains along the equilibrium path, it may also heighten the incentive of an impatient firm to deviate from the collusive agreement. Firms thus choose to avoid communication in some periods and use it to allocate market share in others.

Our finding that firms sometimes, but not always, choose to communicate leads naturally to the question of how collusive behavior changes if anti-trust enforcement prohibits communication. We show that even in the absence of communication, there exists a discount factor strictly less than one above which first-best profits can be achieved. For firms of moderate patience, however, restrictions on communication can diminish collusive profits. In particular, we find that such firms may respond to communication restrictions by reducing productive efficiency without lowering prices. ${ }^{6}$ Thus, our theory offers a formal rationale for the "smoked-filled room" conversations that traditional I.O. associates with collusive ventures; ${ }^{7}$ further, it suggests that anti-trust enforcement that limits such conversations may have perverse welfare implications.

Next, we consider the ability of firms to track firm-level behavior over time, modifying assumption (ii) to suppose that firms face informational or coordination limitations which preclude rewarding an individual firm's current behavior with more favorable treatment in the future. Such limitations arise, for example, when firms interact in an auction market where only the amounts of the bids (but not the identities of the bidders) are publicized. Individual behavior is also difficult to chronicle, if firms can exit and re-enter under different identities, or if participants use agents to conduct business (as is common in auctions).

\footnotetext{
${ }^{6}$ This finding hinges on the assumptions that goods are perfect substitutes and firms compete in prices. Morc generally, restricting communication lowers both prices and productive efficiency.

${ }^{7}$ We may contrast the role of communication in our model with alternative roles identified in the previous literature. First, Compte (1998) and Kandori and Matsushima (1998) consider repeated games in which players have private information about the history of play, arguing that public communication can then constitute a state variable that facilitates the application of recursive techniques. In our model, by contrast, the privatc information is i.i.d. over time and firms' choices (prices) are public, and so the game already has a recursivc structure. Second, McCutcheon (1997) provides an alternative interpretation of communication, involving the rclationship between communication and the renegotiation of the equilibrium. Finally, in the oligopoly information sharing litcrature (e.g., Vives (1984) and Shapiro (1986)), firms commit to share or not share information prior to the play of a static oligopoly game. Incentive compatibility issues do not arise in this literature, as any shared information is, by assumption, directly received or honestly relayed. Sec Kuhn and Vives (1994) for a general survcy of research that explorcs the competitive implications of communication among firms.
} 
Collusion in such environments can be characterized using the solution concept of Symmetric PPE, that is, PPE in which collusion among firms proceeds on an industry-wide basis, so that all firms move through collusive and war phases together. Athey, Bagwell and Sanchirico (1998) provide a more complete analysis of symmetric collusion in a model with a continuum of types and downward-sloping demand. ${ }^{8}$ We confirm here (for a twotype model) two of their findings. First, for a wide range of parameter values, optimal symmetric collusion among sufficiently patient firms is achieved using stationary equilibria, where price wars occur only off of the equilibrium path. Second, in the optimal symmetric equilibria, firms charge the same (high) price irrespective of their cost types, and the cartel sacrifices productive efficiency. Clearly, communication is not necessary for this scheme. We therefore conclude that communication has no value among patient firms, unless the firms are also able to make transfers from one to another as through asymmetric future market-share assignments.

Finally, we examine assumption (iii) by considering environments in which firms can make bribes, though these must be self-enforcing and may incur inefficiencies, as through a risk of detection. Then, firms can potentially substitute current-period bribes for future market-share favors. In practice, monetary transfers may occur directly, with one firm paying other firms for the right to produce. Bribes can be associated with more sophisticated processes, too; for example, colluding suppliers may first hold a "knockout" auction that determines the firm that is to win the procurement contract, and then rig the actual bids to ensure that this firm wins at a profitable price. ${ }^{9}$ We show that when detection by antitrust officials is a concern, so that bribes are not fully efficient, bribes never fully replace future market-share favors as a means of transferring utility. Put differently, unless bribes are perfectly efficient, firms strictly prefer to keep track of history, using non-stationary equilibria that specify a future advantage to firms who admit high cost in the present.

Before proceeding, we pause to place this analysis in the context of recent methodological advances in repeated game theory. We build directly on tools developed by Abreu, Pearce and Stacchetti (1990) and Fudenberg, Levine and Maskin (1994). The latter paper discusses optimal PPE in a repeated game with private information, establishing that an efficient outcome can be achieved when firms are infinitely patient. A novel feature of our analysis is that we characterize optimal PPE with hidden information when firms are impatient. ${ }^{10}$ Within this context, we further show that the optimal scheme can be

\footnotetext{
${ }^{8}$ See also Damania and Yang (1998), who consider a class of symmetric collusion schemes for duopolists with private information as to demand parameters.

${ }^{9}$ Side-payments may also be implemented through a common-fund arrangement, whereby a cartel member that exceeds its production quota contributes a penalty payment to the common fund, while a member that operates below its quota receives a compensatory payment from the common fund. As Stocking and Watkins (1946) describe, arrangements of this general nature appeared in the steel, aluminum and incandescent electric lamp cartels of the carly 1900's.

${ }^{10}$ Independently, Aoyagi (1998) analyzes an auction model where bidders who win in the current period
} 
characterized using tools developed for static mechanism design theory, but where transfers must satisfy particular kinds of restrictions motivated by the shape of the set of PPE continuation values. Thus, in addition to computational analysis, we provide a rich set of theoretical characterizations and comparative statics predictions, while formally highlighting the connections to the static literature. ${ }^{11}$

\section{The Model}

For simplicity, we focus primarily on a highly stylized model with two firms and two cost types. Formally, two firms, 1 and 2, produce perfect substitutes, and sell to a unit mass of customers with valuation $r$. Each firm has possible costs $\theta_{L}$ and $\theta_{H}$ and privately observes its realized costs prior to any pricing decisions. Thus, the state space in any period is denoted - $=\{L, H\} \times\{L, H\}$, and we index these states as $(j, k) \in-$, where the costs of firms 1 and 2 are given by $\theta^{1}=\theta_{j}$ and $\theta^{2}=\theta_{k}$ in state $(j, k)$. The probability of the cost draw $j \in\{L, H\}$ in any period is denoted $\operatorname{Pr}\left(\theta^{i}=\theta_{j}\right)=\eta_{j}$, where $\eta_{j}>0$ and $\eta_{L}+\eta_{H}=1$; this is assumed independent over time and across firms. To simplify the exposition of a few of the results, we maintain the assumption that $\eta_{L}>1 / 2$; however, we will note when this assumption is used.

The Nash equilibrium to the one-shot pricing game (without any communication, commitment or transfer possibilities) is a symmetric mixed strategy equilibrium. ${ }^{12}$ For each firm, the high type charges price equal to cost $\left(p=\theta_{H}\right)$, while the low type mixes, receiving profit equal to $\left(\theta_{H}-\theta_{L}\right) \eta_{H}$, the expected profit from just undercutting the price charged by the high-cost types. Thus, ex ante expected profit to each firm in this equilibrium is equal to $\pi^{N E} \equiv\left(\theta_{H}-\theta_{L}\right) \eta_{H} \eta_{L}$. This payoff can be contrasted with the first-best level of profit to each firm, $\pi^{F B} \equiv \frac{1}{2}\left(r-E\left[\min \left(\theta^{1}, \theta^{2}\right)\right]\right)$.

In our basic repeated-game model, firms can meet and communicate their types but cannot make monetary side-payments. Formally, the firms play the following game in each period: (i) each firm $i$ observes its type $\theta^{i}$; (ii) each firm $i$ makes an announcement $a^{i} \in A \equiv\{L, H, N\}$; (iii) each firm $i$ then selects a price $p^{i}$ and makes a market-share

sit out for a specified number of periods in the future. Athey, Bagwell and Sanchirico (1998) also describe a similar asymmetric scheme. The present paper differs by characterizing optimal asymmetric collusive schemes using dynamic programming.

${ }^{11}$ Our work can be contrasted with the macroeconomics literature (e.g., Green (1987) or Atkeson and Lucas (1992)) on repeated games with hidden information, which studies the game between a central planner and a continuum of agents. Only a few papers consider small numbers of agents (i.e. Wang (1994), and, independently, Cole and Kocherlakota (1998)), and typically the focus of these papers is on existence or computational methods rather than theoretical characterizations.

12 Given that we restrict attention to pure strategy equilibria in the repeated game, the lack of pure strategy equilibria in the stage game creates some tension for the formulation of the model. However, our emphasis on Pareto optimal equilibria implies that in the characterizations we highlight, the best equilibria are pure. 
proposal $q^{i}$; (iv) for $\mathbf{p} \equiv\left(p^{1}, p^{2}\right)$ and $\mathbf{q} \equiv\left(q^{1}, q^{2}\right)$, if $p^{i}>r$, then $q^{i}=0$; if $p^{i}<p^{j} \square r$, then market shares are allocated according to $m^{i}(\mathbf{p}, \mathbf{q})=1$; and if $p^{i}=p^{j} \square r$, then $m^{i}(\mathbf{p}, \mathbf{q})=q^{i}$ if $q^{i}+q^{j} \square 1$, and $m^{i}(\mathbf{p}, \mathbf{q})=\min \left(q^{i}, \max \left(\frac{1}{2}, 1-q^{j}\right)\right)$ otherwise.

This description of the stage game warrants a few comments. We interpret this game as describing an environment in which firms meet, make announcements concerning their respective cost types and then select prices and make market-share proposals. We allow each firm three possible announcements: a firm may announce that it has low costs $(L)$, a firm may announce that it has high costs $(H)$ and a firm may decide not to make an announcement $(N)$. We include the latter option, since, while we allow firms to meet and communicate, they are under no obligation to do so. In our formalization of marketshare proposals, we permit firms to make proposals that jointly determine their respective market shares when they set the same price. Since the market-share proposals follow the firms' announced cost positions, our formalization allows that equally-priced firms may allocate market share in a state-dependent fashion. We can imagine that consumers divide themselves evenly between two equally-priced firms, unless one firm chooses not to sell additional units at the posted price, at which point the consumers go to the other firm. We do not allow, however, both firms to produce positive quantities at different prices. This rules out the possibility that the firms divide the market (for example, geographically) and charge different prices in each segment. ${ }^{13}$

Our next goal is to formally define firm strategies for the stage game. Although the notation is simplified in later sections, for completeness we begin with an abstract representation of these strategies. Letting $-{ }^{i} \equiv\{L, H\}$ and $\mathbf{a} \equiv\left(a^{1}, a^{2}\right)$, we define the space of policies from which a firm might choose as follows:

$$
S^{i}=\left\{\alpha^{i}:{ }^{i} \rightarrow A\right\} \times\left\{\rho^{i}:{ }^{i} \times A \rightarrow \Re\right\} \times\left\{\varphi^{i}:-{ }^{i} \times A \rightarrow \Re\right\} .
$$

A typical policy for firm $i$ is denoted $s^{i}\left(\theta^{i}, a^{j}\right)=\left\{\alpha^{i}\left(\theta^{i}\right), \rho^{i}\left(\theta^{i}, a^{j}\right), \varphi^{i}\left(\theta^{i}, a^{j}\right)\right\}$, where the first component is the announcement function, the second is the pricing function, the third is the market-share proposal function and $a^{j}$ is firm $j$ 's realized announcement. Further, letting $\boldsymbol{\theta} \equiv\left(\theta^{1}, \theta^{2}\right)$, we define the following vectors:

$$
\begin{gathered}
\boldsymbol{\alpha}(\boldsymbol{\theta}) \equiv\left(\alpha^{1}\left(\theta^{1}\right), \alpha^{2}\left(\theta^{2}\right)\right) ; \quad \boldsymbol{\rho}(\boldsymbol{\theta}, \mathbf{a}) \equiv\left(\rho^{1}\left(\theta^{1}, a^{2}\right), \rho^{2}\left(\theta^{2}, a^{1}\right)\right) \\
\boldsymbol{\varphi}(\boldsymbol{\theta}, \mathbf{a}) \equiv\left(\varphi^{1}\left(\theta^{1}, a^{2}\right), \varphi^{2}\left(\theta^{2}, a^{1}\right)\right) ; \quad \mathbf{s}(\boldsymbol{\theta})=\left(s^{1}\left(\theta^{1}, a^{2}\right), s^{2}\left(\theta^{2}, a^{1}\right)\right)
\end{gathered}
$$

\footnotetext{
${ }^{13}$ A benefit of this stage game is that it offers a simple framework (e.g., all transactions occur at the same price and so a rationing rule need not be specified) within which to allow for the possibility that firms communicate and allocate market share in a state-contingent fashion. Of course, other assumptions are possible concerning market-share division and the ability of firms to restrict output and set prices. Our analysis below highlights how the results change if we modify this (somewhat ad hoc) description of market behavior.
} 
Notice that a policy vector $\mathbf{s}(\boldsymbol{\theta})$ determines announcements as well as the price and marketshare proposal responses to these announcements. Accordingly, a policy vector determines a path through the stage game, and we may therefore write stage-game payoffs conditional on a realization of cost types as $\pi^{i}(\mathbf{s}, \boldsymbol{\theta})$, with expected stage-game payoffs then given as $\bar{\pi}^{i}(\mathrm{~s})=E_{\theta}\left[\pi^{i}(\mathrm{~s}, \theta)\right]$.

Now consider notation for the repeated game. The firms meet each period to play the stage game described above, where each firm has the objective of maximizing its expected discounted stream of profit, given the common discount factor $\delta$. As mentioned above, the firms' cost types are i.i.d. across firms and over time, so that the environment is stationary. A novel feature of our model, formally described in the stage game above, is that firms can announce costs types and then set prices and make market-share proposals accordingly. Assume further that, upon entering a period of play, a firm observes only the history of: (i) its own cost draws, and (ii) realized announcements, prices and market-share proposals. Thus, we assume that a firm does not observe rival types or rival policy functions $s^{j}$.

We would like our dynamic game to have a recursive structure, so that dynamicprogramming techniques may be applied. We thus follow Fudenberg, Levine and Maskin (1994) and restrict attention to those sequential equilibria in which firms condition only on the history of realized announcements, prices and market-share proposals and not on their own private history of types and policy schedules. Such strategies are called public strategies and such sequential equilibria are called perfect public equilibria (PPE). Using familiar arguments, we may verify for our game that the continuation of any PPE after any history is itself a PPE in the full game and yields payoffs in the continuation equal to what would have been obtained had the strategy profile been used from the start.

Formally, the public history of realized prices, announcements and market-share proposals up to date $t$ is specified as follows: $h_{t}=\left\{\mathbf{a}_{t}, \mathbf{p}_{t}, \mathbf{q}_{t}\right\}$. Let $H_{t}$ be the set of potential histories at period $t$. A strategy for firm $i$ in period $t$ is denoted $\sigma_{t}^{i}: H_{t} \rightarrow S^{i}$. Let $\sigma_{t}$ be a strategy profile in period $t$, and let $\sigma$ represent a sequence of such strategy profiles, $t=1, \ldots, \infty$. Then, given a history $h_{t}$, the expected per-period payoff in period $t$ for firm $i$ is $\bar{\pi}^{i}\left(\sigma_{t}\left(h_{t}\right)\right)$. Given the mapping from strategies to prices and market shares outlined above, each strategy induces a probability distribution over game play in each period, resulting in an expected payoff for firm $i$, denoted as $v^{i}(\sigma)=E\left[\sum_{t=1}^{\infty} \delta^{t-1} \bar{\pi}^{i}\left(\sigma_{t}\left(h_{t}\right)\right)\right]$, where $h_{1}$ is the null history.

To simplify our analysis, we make a final assumption that is commonly invoked in the literature: we assume that after every period, firms can observe the realization of some public randomization device and select continuation equilibria on this basis. This assumption has the consequence of convexifying the set of equilibrium continuation values available at a particular point in time. While we believe that this assumption is fairly innocuous, convexity of the set of continuation values plays an important role in some 
parts of our analysis. Thus, below (in Section 4.3) we analyze conditions under which convexity obtains without resorting to randomization. For simplicity, we do not introduce explicit notation for the randomization process.

Following Abreu, Pearce and Stacchetti $(1986,1990)$, we can now define an operator $T(V)$ which yields the set of PPE values, $V^{*}$, as the largest invariant, or "self-generating," set. Letting $S \equiv S^{1} \times S^{2}$ and $v \equiv\left(v^{1}, v^{2}\right)$, the operator is defined as follows:

$$
T(V)=\left\{\begin{array}{c}
\left(u^{1}, u^{2}\right): \exists \mathbf{s} \in S \text { and } v: A^{2} \times \Re^{4} \rightarrow c o(V) \text { such that: } \\
\text { for } i=1,2, u^{i}=\bar{\pi}^{i}(\mathbf{s})+\delta E v^{i}(\mathbf{s}(\theta)) \\
\text { and, for each } i \text { and } \tilde{s}^{i}=\left(\tilde{\alpha}^{i}, \tilde{\rho}^{i}, \tilde{\varphi}^{i}\right) \in S^{i}, \\
u^{i} \geq \bar{\pi}^{i}\left(\tilde{s}^{i}, s^{j}\right)+\delta E v^{i}\left[\tilde{s}^{(}\left(\theta^{i}, \alpha^{j}\left(\theta^{j}\right)\right), s^{j}\left(\theta^{j}, \tilde{\alpha}^{i}\left(\theta^{i}\right)\right)\right]
\end{array}\right\}
$$

This operator effectively decomposes equilibrium play into two components, strategies for the current period and continuation values drawn from the convex hull of the set $V$. We record immediately a useful property of $T$ :

Lemma 1. $T$ maps compact sets to compact sets, and the set of PPE values, $V^{*}$, is compact.

Proof. All of the constraints entail weak inequalities; the feasible set is compact; and utility functions and the functions defining the constraints are real-valued, continuous and bounded.

Given compactness, the operator $T$ can also be used to compute the set of equilibrium values, using the following algorithm. Begin with a set $V_{0}$ which is larger than the present discounted value of all possible per-period realizations of utility for each firm. For example, we could take $V_{0}$ to be $[0, r /(1-\delta)] \times[0, r /(1-\delta)]$. Then, construct $V_{n+1}=T\left(V_{n}\right), n \geq 0$. Fudenberg, Levine and Maskin (1994) extend Abreu, Pearce and Stacchetti (1990) to show that $\lim _{n \rightarrow \infty} V_{n}=V^{*}$, the set of equilibrium values $\mathbf{v}(\sigma)$ of the game. We apply this computational algorithm later in the paper.

Our goal is to characterize the operator $T$ and thereby the set of equilibrium values. We are particularly interested in characterizing the Pareto frontier of this set, in order to understand optimal collusive arrangements. From there, it will be possible to back out the strategies that generate the equilibrium values.

To present our findings, we distinguish between two kinds of equilibria, based on the quality of information transmission between firms. In an informative PPE, firms employ equilibrium strategies in which they always share their cost information with one another: for all $i \in\{1,2\}$ and $j \in\{L, H\}$, if $\theta^{i}=\theta_{j}$, then $\alpha^{i}\left(\theta^{i}\right)=j$. As we discuss in more detail in Section 5, this restricts the set of PPE, and we define the operator $T^{I}(V)$ to represent this additional requirement on s. Similarly, in an uninformative PPE, firms are unwilling (or unable) to communicate and make market-sharing plans, and we may capture this situation 
by focusing upon equilibria in which firms never share cost information: for all $i \in\{1,2\}$ and $j \in\{L, H\}, \alpha^{i}\left(\theta_{j}\right) \equiv N$. We use the operator $T^{U}(V)$ to capture this additional requirement on s. Informative and uninformative PPE are of independent interest, and the juxtaposition of these two classes of PPE serves to highlight the benefits and costs of successful information sharing for colluding firms. Furthermore, the characterization of such equilibria contributes to our understanding of the full PPE set, $V^{*}$, since optimal equilibria of the unrestricted PPE class may involve informative communication following some histories and not others.

\section{The Mechanism Design Approach}

In this section, we consider the class of informative PPE and show that the search for the optimal informative PPE can be recast in terms of a static mechanism design program. In addition, we establish the solution to this program in two special cases. The cases correspond to familiar and easily interpretable assumptions in static mechanism design (budget-balanced transfers, no transfers), and they provide useful benchmarks for a more general analysis.

\subsection{Mechanism Notation and Incentive Constraints}

We rely on an application of the revelation principle to simplify our characterization of informative PPE. Since the set of informative PPE values, $V^{I}$, is the largest invariant set of the operator $T^{I}$, every utility vector $\mathbf{u}$ in the set of informative PPE values has associated current-period strategies and continuation-value functions, $s$ and $v$, that yield expected utility of $\mathbf{u}$. When following these strategies, firms report their cost types truthfully and receive the corresponding prices and market-share allocations. Our approach is to introduce notation for such state-contingent prices, market-share allocations and continuation values, and then formalize the corresponding incentive constraints that these must satisfy in order to be implementable as equilibrium play.

We begin with a general description of the incentive constraints. In an equilibrium of the repeated game, there are two kinds of deviations that a firm might entertain. First, a firm with cost type $\theta^{i}$ may consider a deviation in which it adopts the policy that the equilibrium specifies when its cost type is instead $\theta^{i}$ 
(j, ) sn 1 t a s c i n r c $p_{j k}$ prevails. Firm $i$ thereforc sets this price if it makes positive sales (i.e., if $q_{j k}^{i}>0$ ). If firm $i$ makes no sales in state $(j, k)$, then firm $i$ 's price may differ from $p_{j k}$, but it cannot be lower. 


$$
U^{i}(L, L ; \mathbf{z}) \geq U^{i}(H, L ; \mathbf{z})
$$

We next formally represent the incentive that a firm has to charge a price not assigned to any cost type, that is, to undertake an off-schedule deviation. In an informative PPE, there are two types of off-schedule incentive constraints. The first concerns the incentive of a firm to deviate from the assigned price after communication takes place. If both firms are assigned a price less than firm 1's cost, firm 1 might like to price slightly above firm 2 , to avoid producing in that state; alternatively, at higher prices, firm 1 might wish to slightly undercut firm 2's price and capture the entire market. ${ }^{16}$ If the following constraint is satisfied, neither of these deviations is profitable:

$$
\delta\left(v_{j k}^{1}-\underline{v}^{1}\right) \geq \max \left(q_{j k}^{2}\left(p_{j k}-\theta_{j}\right), q_{j k}^{1}\left(\theta_{j}-p_{j k}\right)\right) .
$$

where $\underline{v}^{i}=\underline{v}^{i}(V) \equiv \inf \left\{v^{i}: v \in V\right\}$. (We will write $\underline{v}^{i}$ rather than $\underline{v}^{i}(V)$ to conserve notation, and take the off-schedule constraints relative to the set of values under consideration in a particular context.) As $\underline{v}^{i}$ is reached only off of the equilibrium path, we can essentially treat it as a parameter in the analysis. IC-Off2 ${ }_{j k}^{I}$ is defined analogously.

It is worth pausing to notice how the form of these constraints follows from our assumptions about communication and timing. The constraints must hold state by state, since when communication among firms is informative each firm knows when setting its price the cost type (and thus the price) of its opponent. These constraints can be contrasted with the case without informative communication (analyzed in more detail below), whereby an off-schedule price deviation is contemplated without knowledge of a rival's cost. Of course, other assumptions about timing are also possible. ${ }^{17}$

The second type of off-schedule deviation is an interim deviation, described as follows. Suppose that the collusive scheme assigns a lower price in state $(L, L)$ or $(L, H)$ than in $(H, H)$ or $(H, L)$. If firm 1 draws a low cost, firm 1 might be tempted to report a high cost, in order to induce firm 2 to price high, so that firm 1 might then undercut firm 2's high price. Such an undercut might be attractive for exactly one of firm 2's cost types, or perhaps for both, and firm 1 can wait to learn the realization of firm 2's type before making a final decision to undercut. Deviations of this kind are dissuaded if:

$$
U^{1}(L, L ; \mathbf{z}) \geq \sum_{k \in\{L, H\}} \eta_{k} \cdot \max \left(q_{H k}^{2}\left(p_{H k}-\theta_{L}\right)+\delta \underline{v}^{1}, q_{H k}^{1}\left(p_{H k}-\theta_{L}\right)+\delta v_{H k}^{1}\right), \text { (IC-Off-M1) }
$$

${ }^{16}$ Given that unit costs are constant in output, a firm best deviates by claiming all market share or relinquishing all market share. In either event, a small change in price serves the purpose. We therefore need not concern ourselves with the possibility that a firm deviates by maintaining the price and adjusting up or down its proposed market share.

17 For example, if the firms are bidding in an auction where only one firm can win, they might use a randomization device to select the winner, where $q_{j k}^{i}$ is the probability $i$ wins in state $(j, k)$; then, the onschedule constraints would be identical, but the off-schedule constraints would require that unless $q_{j k}^{i}=1$, player $i$ must be willing to "sit out" entirely if the randomization turns against her. We do not analyze this more-restrictive alternative, but the qualitative results are similar. 
where the $\mathrm{M}$ is mnemonic for "misrepresentation." The constraint for firm 2 is defined analogously. It can be easily verified that, so long as IC-Off $1_{L k}^{I}$ is satisfied for $k \in\{L, H\}$, the high type never has the incentive to engage in this type of misrepresentation. Further, it is clear that (as will be the case in many of our characterizations below) if prices are the same in each state, this type of deviation is (weakly) dominated.

\subsection{The Repeated Game as a Mechanism}

We introduce notation for the feasible set of policy vectors when firms use informative communication, given an arbitrary set of continuation values $V$.

$$
\mathcal{F}^{I}(V)=\left\{\begin{array}{c}
\mathbf{z}=(\mathbf{p}, \mathbf{q}, \mathbf{v}) \in \mathcal{Z}(V): \text { For all } i=1,2, \\
\text { IC-On } i_{D}, \text { IC-On } i_{U}, \text { IC-Off } i_{j k}^{I} \text { and IC-Off-M } i \text { hold. }
\end{array}\right\}
$$

With this notation in place, we present the following lemma.

Lemma 2. Given a set $V \subset \Re^{2}$, let

$$
\tilde{T}^{I}(V)=\left\{\begin{array}{c}
\left(u^{1}, u^{2}\right): \exists \mathbf{z}=(\mathbf{p}, \mathbf{q}, \mathbf{v}) \in \mathcal{F}^{I}(V) \\
\text { such that for } i=1,2, u^{i}=\bar{U}^{i}(\mathbf{z}) .
\end{array}\right\}
$$

Then $\tilde{T}^{I}(V)=T^{I}(V)$.

The lemma follows by a comparison of constraints and an application of standard revelation principle arguments. Intuitively, once we have invoked the tools of dynamic programming to decompose the problem into a choice of current-period strategies and future continuation equilibria, the revelation principle applies to the "staticized" representation of the game. For the class of informative PPE, Lemma 2 formalizes the relationship between the repeated game and the mechanism design problem we have just defined. It states that we can characterize the operator $T^{I}$ as generating the set of all utilities that satisfy the constraints of a fairly standard mechanism design problem, with the addition of the unusual restriction $\left(v_{j k}^{1}, v_{j k}^{2}\right) \in V$.

An important consequence of this result is that for any informative PPE utility vector $\mathbf{u}$, there exists a policy vector $(\mathbf{p}, \mathbf{q}, \mathbf{v})$ th

ts $V$. These examples are motivated by the static mechanism design literature where $V$ is the set of available monetary transfers. In the first example, $V$ is a line of slope -1 ; this represents "budget-balanced" transfers of utility that incur no efficiency loss. In the second example, 
we consider sets of the form $V=\left\{\left(v^{1}, v^{2}\right): v^{1}, v^{2} \square K\right\}$; for such sets, all continuation values except $(K, K)$ are Pareto inefficient. The cases are illustrated in Figure 1. These benchmarks allow us to develop some basic intuition, on which we build when we later consider sets $V$ with more general shapes, such as the convex set illustrated in Figure 2.

To draw most clearly the analogy to the static mechanism design literature, we ignore the off-schedule incentive constraints in this section. We then refer to the set of constraints excluding off-schedule incentive constraints as $\mathcal{F}_{O n}^{I}(V)$, and we define:

$$
\tilde{T}_{\text {On }}^{I}(V)=\left\{\begin{array}{l}
\left(u^{1}, u^{2}\right): \exists \mathbf{z}=(\mathbf{p}, \mathbf{q}, \mathbf{v}) \in \mathcal{F}_{O n}^{I}(V) \\
\text { such that for } i=1,2, u^{i}=\bar{U}^{i}(\mathbf{z}) .
\end{array}\right\}
$$

In discussing schemes, we say that a scheme uses productive efficiency if for every state $(j, k) \in-, q_{j k}^{1}+q_{j k}^{2}=1$ and $q_{L H}^{1}=q_{H L}^{2}=1$. We say that a scheme uses efficient pricing if $p_{j k}^{i}=r$ for all $(j, k) \in$-. Similarly, the scheme is characterized by Pareto efficient continuation values if for every $(j, k)$, there does not exist a continuation value pair $\left(\tilde{v}^{1}, \tilde{v}^{2}\right) \in V$ that Pareto dominates $\left(v_{j k}^{1}, v_{j k}^{2}\right)$.

To begin, we record the following standard lemma:

Lemma 3. Any z satisfying IC-Oni $i_{D}$ and $I C-O n i_{U}$ also satisfies $\bar{q}_{H}^{i} \square \bar{q}_{L}^{i}$. If IC-Oni ${ }_{D}$ binds, then

$$
U^{i}(H, H ; \mathbf{z})=U^{i}(L, H ; \mathbf{z})=U^{i}(L, L ; \mathbf{z})-\bar{q}_{L}^{i}\left(\theta_{H}-\theta_{L}\right)
$$

Market-share monotonicity follows since our model satisfies a single-crossing property: the low-cost type has a higher marginal return to market share. The representation of the relationship between the expected utility of each type follows by algebraic manipulation; it says that the low-cost type earns an "efficiency rent" of $\bar{q}_{L}^{i}\left(\theta_{H}-\theta_{L}\right)$ over the high-cost type. It also illustrates a discrete-type variation on the classic "revenue equivalence theorem": when IC-On $i_{D}$ binds for each firm, then the ex ante expected utility for firm $i$ is given by

$$
\bar{U}^{i}(\mathbf{z})=U^{i}(H, H ; \mathbf{z})+\eta_{L} \bar{q}_{L}^{i}\left(\theta_{H}-\theta_{L}\right)=\Pi^{i}(H, H ; \mathbf{z})+\delta \bar{v}_{H}^{i}+\eta_{L} \bar{q}_{L}^{i}\left(\theta_{H}-\theta_{L}\right) .
$$

This reveals that among the set of allocation rules where IC-On $i_{D}$ binds, firm $i$ is indifferent between providing incentives with low prices or low continuation values for its low-cost type. This follows because (in contrast to market share) neither the price nor the continuation value interacts directly with the firm's type in the firm's objective function. Thus, the cartel has a preference over the use of low-cost prices and continuation values for which a firm's on-schedule incentive constraint binds, only insofar as these instruments generate efficiency losses or gains for the other firm. Lowering price decreases the utility of both firms. In contrast, when cross-firm transfers of utility are available, lowering one firm's continuation value may allow an increase in that of the other firm, and continuation values are then a superior instrument for maintaining on-schedule incentives. 
To better highlight some of these themes, we turn now to two special cases. First, we suppose that the set of feasible continuation values is a line of slope $-1 .^{18}$

Lemma 4. For $K \in \Re$, suppose that $V(K)=\left\{\left(v^{1}, v^{2}\right): v^{1}+v^{2}=2 K\right\}$. Then, for any $K \geq 0$, the Pareto frontier of $\tilde{T}_{O n}^{I}(V(K))$ is $\left\{\left(u^{1}, u^{2}\right): u^{1}+u^{2}=\delta 2 K+2 \pi^{F B}\right\}$, and this frontier can be implemented with a policy vector $\mathbf{z}$ that satisfies the following properties: productive efficiency, pricing efficiency and Pareto efficient continuation values $\left(v_{j k}^{1}+v_{j k}^{2}=2 K\right.$ for all $\left.j, k\right) ; v_{H L}^{1}-v_{L H}^{I}=\left(r-\theta^{H}\right) / \delta$; the downward $I C$-On constraints bind; the upward IC-On constraints are slack; and $v_{L H}^{1}<v_{j j}^{1}<v_{H L}^{1}$ for $j \in\{L, H\}$.

This result merits some interpretation. As expected, first-best is attained. The downward IC-On constraints bind, since it is the low-cost type who has the higher market share, and market share is desirable for both firms. Thus, the relevant consideration is to dissuade the high-cost type from mimicking the low-cost type; as lower cost types have a higher marginal benefit to high market share, if the high-cost type is just indifferent between the high and low announcement, the low-cost type strictly prefers the low-cost announcement. The optimal mechanism requires transfers through continuation values that reward a firm for announcing high costs. In particular, when the policy vector satisfies productive, pricing, and continuation-value efficiency, and the continuation values lie on a budget-balanced line, the IC-On $i_{D}$ constraints bind if and only if $v_{H L}^{1}-v_{L H}^{1}=\left(r-\theta^{H}\right) / \delta$, which when discounted equals the maximum amount that a high-cost firm could gain by misreporting.

Next, we consider a second special case, wherein the firms receive continuation values from a set in which each firm can receive at most $K$. To state the result, we refer to the following condition:

$$
\left(r-\theta_{H}\right) /\left(\theta_{H}-\theta_{L}\right)>\eta_{H}
$$

Lemma 5. Suppose that $V(K)=\left\{\left(v^{1}, v^{2}\right): v^{1}, v^{2} \square K\right\}$. (i) Suppose that (Pool) holds. Then, for any $K$, the Pareto frontier of $\tilde{T}_{O n}^{I}(V(K))$ is

$$
\left\{\left(u^{1}, u^{2}\right): u^{1}+u^{2}=r-E[\theta]+\delta 2 K, u^{i} \geq 0\right\}
$$

and this frontier can be implemented with a policy vector $(\mathbf{p}, \mathbf{q}, \mathbf{v})$ that satisfies the following properties: pricing efficiency, Pareto efficient continuation values $\left(v_{j k}^{i}=K\right.$ for all $i, j, k$ ) and productive inefficiency with $\bar{q}_{H}^{i}=\bar{q}_{L}^{i}$ for $i=1,2$. (ii) Suppose that (Pool) fails. Then the Pareto frontier of $\tilde{T}_{O n}^{I}(V(K))$ is given by

$$
\left\{\left(u^{1}, u^{2}\right): u^{1}+u^{2}=\eta_{H}\left(r-\theta_{H}\right)+\eta_{L}\left(1+\eta_{H}\right)\left(\theta_{H}-\theta_{L}\right)+\delta 2 K, u^{i} \geq 0\right\} .
$$

\footnotetext{
${ }^{18}$ For the public goods problem with private information, d'Aspremont and Gerard-Varet (1979) show that the first-best can be attained using budget-balanced transfers, when the mechanism must satisfy incentive compatibility but not participation constraints. McAfee and McMillan (1992) specialize this result for the case of first-price auctions, showing that participation constraints in fact can be satisfied in this problem. The following result is a further specialization to the two-type model, rephrased to allow for continuation values to sum to a constant other than zero.
} 
his can be implenented using productive efficiency, Pareto efficient continuation values $\left(v_{j k}^{i}=K\right.$ for all $\left.i, j, k\right)$, pricing efficiency in state $(H, H)\left(p_{H H}=r\right)$ and a price of $\frac{\eta_{H}}{1+\eta_{H}}(r-$ $\left.\theta_{H}\right)+\theta_{H}$ in the remaining states.

Lemma 5 refers to an environment in which the only instruments available (reduced continuation values, low prices) with which to achieve incentive compatible productive efficiencies are wasteful. When (Pool) holds, so that the profit to the high-cost type is large relative to the efficiency advantage of the low-cost type, Lemma 5 establishes that the Pareto frontier entails productive inefficiency: the loss in profit from either Pareto inefficient continuation values or inefficient pricing then overwhelms any potential productive efficiency gain.

To see the logic of the condition (Pool), consider raising productive efficiency by increasing $q_{H L}^{2}$ (and therefore decreasing $q_{H L}^{1}$ ). The subtle aspect of the intuition entails understanding the effects of this change when prices and continuation values must adjust to maintain the on-schedule incentive constraints. The change decreases firm 1's ex ante expected utility by $\eta_{L}\left(r-\theta_{H}\right)$, since firm 1's high type bears the cost directly and firm 1's low type must now charge a lower price or receive a lower continuation value to avoid violating IC-On $1_{D}$. The change increases firm 2's ex ante expected utility by $\eta_{L} \eta_{H}\left(\theta_{H}-\theta_{L}\right)$, the higher "efficiency rent" $\left(\theta_{H}-\theta_{L}\right)$ available to firm 2's low-cost type in state $(H, L)$. (Pool) guarantees that the cost to firm 1 , incurred across both states $(H, L)$ and $(L, L)$, outweighs the efficiency benefit to firm 2 in state $(H, L)$.

This result introduces a theme that will recur throughout our analysis. It illustrates that there is a "tax" on productive efficiency: improving productive efficiency tightens the on-schedule incentive constraint, leading to further distortions.

If, in contrast, $\left(r-\theta_{H}\right) /\left(\theta_{H}-\theta_{L}\right)<\eta_{H}$, the firms prefer to incur the incentive costs that the achievement of productive efficiency requires. These costs may be manifested as low continuation values or low prices for the low-cost types. In the environment under consideration, the firms are indifferent between these two alternatives, and in particular, it is always possible to achieve the optimal collusive payoffs using the highest available continuation values and low prices for the low-cost types. Further, notice that the pricing scheme outlined in the lemma can be implemented decentrally: each firm charges a price of $r$ when its own cost is high, and selects a price $\hat{p}$ when its own cost is low. This allocates market share efficiently and achieves the price of $\hat{p}$ in all states except $(H, H)$.

Whether firms choose to produce efficiently or not, expected profit to a cartel is not improved by moving from a situation where $V=\{(K, K)\}$ to a situation where $V=$ $\left\{\left(v^{1}, v^{2}\right): v^{1}, v^{2} \square K\right\}$. In other words, wasteful continuation values are not useful as instruments for providing incentives. In a repeated game context, this implies that if the set of equilibrium values is restricted to take the form $V=\left\{\left(v^{1}, v^{2}\right): v^{1}=v^{2}\right\}$, as in the case of Symmetric PPE, optimal equilibria are stationary: "price wars" are not employed. 
We elaborate on this result further in Section 6. More general oligopoly models also have similar forces present: the on-schedule incentive constraints create cross-type externalities that favor pooling of cost types. ${ }^{19}$

This result can be related to the existing literature, where types are typically taken continuous, in several ways. In their analysis of "weak cartels," McAfee and McMillan (1992) show that when transfers are prohibited $(V=\{(0,0)\})$, and the distribution over types, $F(\theta)$, is log-concave, the best collusive scheme entails all firms pricing at the reservation price. Athey, Bagwell and Sanchirico (1998) extend this result to the case where $V=\left\{\left(v^{1}, v^{2}\right): v^{1}=v^{2}\right\}$, as motivated by Symmetric PPE. The continuum-type approach and our two-type approach can be most easily connected with reference to an $N$-type model. Let $\eta_{n}$ be the probability that cost type $n$ is realized. Then, the following conditions replace (Pool): $\left(r-\theta_{N}\right) \eta_{m}-\eta_{N} \sum_{n=1}^{m-1} \eta_{n}\left(\theta_{n+1}-\theta_{n}\right)>0$ for all $m$; and $\sum_{n=1}^{m-1} \eta_{n}\left(\theta_{n+1}-\theta_{n}\right) / \eta_{m}$ is nondecreasing in $m$. The first expression is the analog of (Pool); the second condition is the analog of log-concavity of the distribution $F(\theta)$.

\section{Characterization of Informative PPE}

In this section, we characterize the set of informative PPE values. Our analysis builds on the insights developed in the benchmark cases of Section 3.3. Throughout, we develop analytically some key findings, and we then illustrate additional subtleties with computational examples.

Before beginning the formal analysis, we outline some of the central tradeoffs. Suppose that the firms attempt to implement first-best profits. Consider how this might be accomplished, and the factors that might prevent the firms from reaching this goal. In the first period of the game, a first-best scheme must implement productive efficiency and pricing efficiency; thus, from the perspective of current-period profits, high-cost firms are tempted to mis-report their costs in order to achieve greater market share. To ensure truthful reporting, the agreement therefore must provide that firm 2 receives future market-share favors from firm 1 following a realization of the state $(L, H)$. Suppose then that $(L, H)$ is realized in the first period, and consider the scheme in the second period of the game. In a first-best collusive scheme, productive efficiency is again required; consequently, if state $(L, H)$ is once more realized, then firm 2 must again receive zero market share. On the other hand, if the firms experience the same costs in the second period, then the collu-

\footnotetext{
${ }^{19}$ When the monopoly price varies with the cost type, firms seek to tailor the price to the cost realization. For the case of Symmetric PPE, Athey, Bagwell and Sanchirico (1998) show that if demand is sufficiently downward-sloping, the firms find it optimal to partially sort types. In a two-type model, if (Pool) holds, optimal schemes entail productive efficiency only if the cost types are sufficiently different and the slope of the demand function is large enough (or if the firms are too impatient to support the high prices of the collusive equilibrium).
} 
sive arrangement may favor firm 2 while simultaneously delivering first-best profits. This is achieved by giving firm 2 more than $1 / 2$ of the market in the second period when the $(L, L)$ and $(H, H)$ states are realized. If these market shares are appropriately chosen, both firms still have the incentive to report truthfully.

What might prevent such a scheme from succeeding? The firms must be sufficiently patient so that firm 1 is dissuaded from undertaking an off-schedule deviation following a realization of $(L, L)$, when its assigned market share is low. What if this cannot be accomplished? Then, asymmetric treatment introduces new inefficiencies. In particular, the scheme may require low prices, or it may call upon the disadvantaged firm to relinquish some market share in the state in which it is most efficient, as its temptation to undertake an off-schedule deviation is low when its assigned market share is high.

Pulling these themes together, we may summarize the central tradeoffs as follows. If in a given period, the firms seek productive efficiency "today," then asymmetric treatment is required "tomorrow." Productive and pricing efficiency tomorrow, however, can then be maintained only if the asymmetric treatment is implemented through asymmetric marketshare assignments among equally-efficient firms pricing at the reservation value. In turn, this is possible only if tomorrow the disadvantaged firm is sufficiently patient to endure its assigned low market share. In view of these tradeoffs, a cartel comprised of moderately patient firms may assign market shares today without achieving full productive efficiency, in order to lessen the future transfer burden.

In the next two subsections, we derive conditions on the discount factor under which firms are able to implement a given level of efficiency (such as first-best) in every period of the game. Subsequently, we explore in greater depth the optimal resolution of the tradeoffs between current and future efficiency faced by firms of moderate patience.

\subsection{A Linear Informative PPE Set With First-Best Profits}

In this subsection, we identify a discount factor strictly less than one above which the cartel can achieve first-best profits in every period. Recall that Section 3.3 analyzes Pareto optimal schemes for an exogenously given set of continuation values. We now confront directly the endogenous nature of the continuation-value set. Our goal is to establish the existence of a set of Informative PPE values, where (i). each utility pair yields first-best profits to the cartel as a whole, and (ii). when implementing any point in the set, only other elements of the set are used as continuation values on the equilibrium path (by contrast, off of the equilibrium path, punishment outside the set is required.) Clearly, a "self-generating" set comprised only of values that yield first-best profits must be a line segment with slope -1 .

We seek to construct a self-generating line segment with endpoints denoted $(x, y)$ and $(y, x)$. The first step is to ignore off-schedule constraints and identify conditions under 
which the endpoint $(x, y)$ can be implemented using only continuation values on the line segment. ${ }^{20}$ Formally, we explore conditions under which there exist market shares $q_{H H}^{1}$, $q_{L L}^{1} \in[0,1]$, as well as continuation values $v_{j k}^{1} \in[x, y]$ with $v_{j k}^{2}=x+y-v_{j k}^{1}$, such that $(x, y)=\left(\bar{U}^{1}(\mathbf{z}), \bar{U}^{2}(\mathbf{z})\right)$ and IC-On $i_{D}$ binds for each $i$, when the firms use pricing and productive efficiency.

This step can be challenging for certain parameter values. If monopoly profit for a highcost firm, $r-\theta_{H}$, is too large, it may be difficult to achieve the desired level of profit for firm $1, \bar{U}^{1}(\mathbf{z})=x$, while maintaining $v_{j k}^{1} \geq x$. To see why, observe that firm 1's average profit today must then be worse than its per-period profits derived from each of the continuation values: $E\left[\Pi^{1}(j, j ; \mathbf{z})\right] \square v_{j k}^{1}(1-\delta)$ for each $(j, k)$. Further, in order to provide incentives for firm 1 to reveal its type when it is high cost, the future must look relatively better following a realization of $(H, L)$ : following the logic of Lemma 4 , a necessary condition for each IC-Oni $i_{D}$ to bind is that $v_{H L}^{1}-v_{L H}^{1}=\left(r-\theta_{H}\right) / \delta$, and so $v_{H L}^{1}$ must exceed $x$ by at least $\left(r-\theta_{H}\right) / \delta$. This requirement places additional downward pressure on today's expected profit, which must be low enough to satisfy $\bar{U}^{1}(\mathbf{z})=x$. However, productive and pricing efficiency impose a lower bound on today's profit. Similarly, if the efficiency-rent term, $\theta_{H}-\theta_{L}$ is too small, it can be difficult to implement $\bar{U}^{2}(\mathbf{z})=y$ while maintaining $v_{j k}^{2} \square y$. Intuitively, firm 2's average profit today must then be greater that its per-period profits derived from each of its continuation values. Recalling (3.2), this is more easily achieved when the efficiency rent $\theta_{H}-\theta_{L}$ is large, as then current-period profit for firm 2 can be significantly increased by raising $q_{L L}^{2}$, while still respecting the on-schedule incentive constraints.

In the Appendix, we show that there exists a critical discount factor less than 1 where the constraints listed above are all satisfied, if (recalling our assumption $\eta_{L}>1 / 2$ ): ${ }^{21}$

$$
\eta_{L}^{2}>\frac{r-\theta_{H}}{\theta_{H}-\theta_{L}}\left(2 \eta_{L}-1\right)
$$

We may verify that (4.1) is satisfied if $r-\theta_{H}<\theta_{H}-\theta_{L}$; more generally, it holds if $\eta_{L}$ is sufficiently close to $1 / 2 .{ }^{22}$ Under (4.1), we show in the Appendix that implementation is

${ }^{20}$ We argue in Section 4.3 that if $\mathrm{z}$ and $\mathrm{z}$

$\square y$. Although we do not formally analyze this case, it can be shown that for $\eta_{L}<\frac{1}{2}$, a different but analogous condition must hold.

${ }^{22}$ If this parametric restriction fails, then the firms may not be able to implement exactly first-best profits. However, it is possible to construct self-generating sets composed of three connected line segments, where the interior line segment has slope -1 , and all points on that segment are implemented using productive efficiency. In contrast, some productive inefficiency is used on the exterior segments. As firms become more patient, the width of the interior line segment grows, and so first-best can be approximated arbitrarily closely as $\delta$ approaches 1 . 
feasible if $\delta$ is greater than a critical discount factor, $\delta^{\text {Fon }}$, defined as follows:

$$
\delta^{F o n}=\frac{r-\theta_{H}}{\left(\theta_{H}-\theta_{L}\right) \eta_{L}^{2}+2\left(r-\theta_{H}\right)\left(1-\eta_{L}\right)}
$$

The second step is to identify a second critical discount factor, above which the offschedule incentive constraints hold. It is sufficient for our construction to specify that an off-schedule deviation leads to an infinite reversion to the static Nash equilibrium. As is standard, if firms are sufficiently patient, then an off-schedule deviation is unattractive. In the Appendix, we describe the computation of the critical discount factor, $\delta^{F o f f}$, above which the off-schedule constraints are cleared: ${ }^{23}$

$$
\delta^{O f f}=\frac{\left(r-\theta_{H}\right)\left(1-\eta_{L}\right)+\eta_{L}\left(\theta_{H}-\theta_{L}\right)}{\left(r-\theta_{H}\right)\left(1-\eta_{L}\right)+\eta_{L}\left(\theta_{H}-\theta_{L}\right)+\eta_{L}^{2}\left(r-\theta_{H}\right)} .
$$

Proposition 1. Suppose that $r-\theta_{H}<\theta_{H}-\theta_{L}$, and define $\delta^{F B}=\max \left(\delta^{F o n}, \delta^{F o f f}\right) \in(0,1)$. Then, for all $\delta \in\left(\delta^{F B}, 1\right]$, there exist values $y>x>0$ such that $x+y=2 \pi^{F B} /(1-\delta)$, and the line segment $[(x, y),(y, x)]$ is in the set of informative PPE values, $V^{I}$. Each utility pair $\mathbf{u}$ on the segment can be implemented using a policy vector $(\mathbf{p}, \mathbf{q}, \mathbf{v})$ such that pricing and productive efficiency hold, and $v_{j k} \in[(x, y),(y, x)]$ for each $(j, k)$.

For the computational example we explore below, where $r=2.5, \theta_{H}=2, \theta_{L}=1$ and $\eta_{L}=.6$, we find that $\delta^{\text {Fon }}=.66$, and $\delta^{\text {Foff }}=.82$.

Proposition 1 can be thought of as a generalization of Fudenberg, Levine and Maskin's (1994) folk theorem. Instead of resorting to infinite patience, we compute a discount factor strictly less than one (.82 in the previous example) where first-best is achieved. Our result further provides an explicit characterization of the behavior associated with this first-best arrangement: since real inefficiencies might be associated with the provision of incentives, and since some firms may be at the margin of deviating from the agreement, it is important that the cartel design the collusive agreement to circumvent these issues. The cartel achieves this by shifting market share among firms in states where productive efficiency is not sacrificed. ${ }^{24}$

23 This discount factor may be conservative, since it is computed using a particular method of implementing the self-generating set, one that applies for all parameter regions under consideration. For particular parameter regions, there may alternative self-generating sets that can be implemented with lower levels of patience.

${ }^{24}$ It is useful to contrast our characterization of collusive behavior with themes from the review-strategy literature (Radner, 1981) as developed in "hidden action" games with infinitely-patient firms. Under this approach, after many periods, there is a "review," whereby the distribution of observables is compared to the expected distribution if each firm had followed the specified strategy. Any firm whose behavior over that period of time does not conform is punished as harshly as possible. In the limit, patient firms view the small probability of punishment as sufficiently bad that they conform to the required strategies. Further, the review periods can be taken to be arbitrarily distant, so that in the limit, punishments occur with zero probability. In our model, by contrast, firms are not infinitely patient, and so real inefficiencies may be required to provide incentives. Our analysis is concerned with the optimal manner in which to provide such incentives. 


\subsubsection{Computational Example}

Throughout Section 4, we use a computational example to develop our insights in further detail. We now offer some general remarks about our computational approach, and we next consider an explicit parameterization that relates to Proposition 1.

Our approach is to specify a set $V^{0}$ and then compute $V^{t}=\tilde{T}^{I}\left(V^{t-1}\right)$ for $t=1, \ldots$, iterating until the distance between the sets becomes lower than a given tolerance level. To operationalize this algorithm, a natural method is to divide each set $V^{t}$ into a grid, and then check which members of this grid survive to become members of $V^{t+1}$. This approach is slow, however. Following Wang (1994), we use a trick which speeds up the computations. ${ }^{25}$ At the start of the algorithm, we divide the set $[0, r /(1-\delta)]$ into a fixed grid, where we let $\mathbf{x}$ denote the vector of points in this grid. The grid represents the set of feasible continuation values for firm 2 , and these are the only values ever permitted for firm 2 . On each iteration of the algorithm, we compute the best and worst continuation values for firm 1 which can be sustained for each $x_{k}$ in this grid.

To further ease the computational burden, we impose two restrictions. First, rather than compute the lower bound of the set $V^{t}$ on each iteration, we approximate this lower bound with static Nash equilibrium payoffs. The restriction to static Nash punishments does not directly affect the qualitative characterization of the efficiency frontier, since (as the computations show) the firms only leave the efficiency frontier off of the equilibrium path. The available punishment, however, does affect the extent to which firms can sustain asymmetric equilibrium values. Thus, it indirectly affects the continuation values for every point on the frontier. Second, we restrict the firms to use pricing efficiency. ${ }^{26}$ Given the restrictions we have imposed, our computations should be interpreted as lower bounds on the Pareto frontier of equilibria.

Figures 3,4 and 5 illustrate our computation for a particular set of parameter values. We number the states on the frontier. The table below the figure shows how the game proceeds from every point on the frontier. Observe that the computations yield slightly asymmetric continuation values across the two firms; this arises as a result of the computational algorithm, which treats one firm's profit as discrete and the other's as continuous. Further, on the region where the continuation value frontier is approximately linear, there are often many ways to implement a given value; but, due to the discretization of the frontier, the firms may have a strict preference among alternative collusive schemes giving

${ }^{25}$ Wang's (1994) approach builds in turn on Phelan and Townsend (1991). Recently, Judd (1995) has developed several approaches to computation that could be substantially more efficient. As our aim with the computations is to illustrate the theoretical results, we have not pursued this approach, though it is an interesting avenue for future work.

${ }^{26}$ For impaticnt firms, this restriction is perhaps more onerous; we show in later subsections that for some parameter valucs, even if (Pool) holds, pricing inefficiency may be desirable on the Pareto frontier when the off-schedule incentive constraints bind. 
approximately the same utility. Thus, occasionally behavior appears to "jump" drastically among nearby states. This does not qualitatively affect the computation of the equilibrium set.

Using the table of transitions, it is possible to trace the path of play given any sequence of cost realizations, taking any state on the Pareto frontier as the starting point. The market-share table illustrates the extent of productive efficiency in each state.

Figure 3 illustrates the implementation of an equilibrium yielding first-best profits for the cartel at every point in time. The region of states between 11 and 24 is self-generating ${ }^{27}$, and productive efficiency is used (approximately) in each state. Incentives for truthful revelation of the cost type are provided using continuation values, with $v_{H L}^{1}-v_{L H}^{1}=$ $\left(r-\theta_{H}\right) / \delta$.

It is particularly useful to consider the implementation of the equilibrium value corresponding to state 11 , since the off-schedule constraints for the extreme values of the linear self-generating set are the most demanding. Consistent with the theoretical analysis above, we see that in state 11, firm 2 produces (essentially) only in state $(H, L)$, where it is most efficient. Further, following the cost realization $(H, L)$, the equilibrium specifies that the firms return to state 11 . In contrast, following all other cost realizations, firm 2 does not produce, but does receive a more favorable continuation value. In other words, larger "rewards" accrue to firm 2 when it announces high cost, while the future "punishment" following the realization $(H, L)$ entails remaining in state 11. Clearly, firm 2 must be relatively patient to endure sitting out when the cost realization is $(L, L)$, but the prospect of moving to state 15 in the following period induces firm 2 to adhere to the agreement. In contrast, when implementing state 7 , firm 2 cannot adhere to the agreement if its equilibrium profit following a realization of $(L, L)$ is any lower; thus, the only way to lower payoffs to firm 2 is to lower firm 2's market share in state $(H, L)$, where the off-schedule constraints are slack since firm 2's market share is high.

\subsection{A Linear Informative PPE Set with Partial Productive Efficiency}

As mentioned above, when firms are less patient, their ability to use market-share favors in future "tie" states is limited. The remainder of Section 4 analyzes collusive schemes in such circumstances. Although our main focus is on characterizing the Pareto frontier of the Informative PPE set, before proceeding in that direction, we briefly extend the approach of the last section to consider linear self-generating sets of Informative PPE values, where only partial productive efficiency is used. Even though such sets typically are not Pareto optimal, we analyze them for two reasons. First, in contrast to the Pareto frontier, which often must be approximated numerically, linear self-generating sets are tractable to analyze

\footnotetext{
${ }^{27}$ It may be verified that self-gencrating sets are not unique: the regions of states between 8 and 24,10 and 24,12 and 24 , and 13 and 24 , respectively, are also self generating.
} 
in closed form. Second, a self-generating set of equilibrium values provides a lower bound on the level of profits that can be sustained by colluding firms in an optimal PPE. This lower bound, phrased in terms of exogenous parameters, is useful when we attempt to characterize the Pareto frontier in the next section.

Depending on parameter values, a variety of different constraints may bind in the implementation of a linear self-generating set. In this section, we take a conservative approach, constructing a linear set $[(x, y),(y, x)]$ where the off-schedule constraints bind in two states for firm 1 when implementing $(x, y)$. To construct the line segment, we proceed by specifying a set of constraints that bind, and we then verify that the others are satisfied. Formally, suppose that, when implementing $(x, y)$, the following constraints bind:

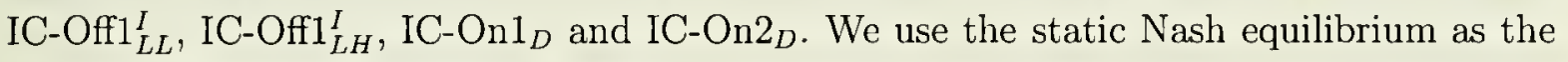
threat point in the off-schedule constraints. Further, we specify that $p_{j k}=r$ for all $(j, k)$, $v_{L H}=v_{L L}=(x, y), v_{H H}=v_{H L}$, and $q_{H L}^{1}=q_{H H}^{1}=0$. It can be verified that under these restrictions, $q_{L L}^{1}=q_{L H}^{1} \in(0,1)$. It remains to verify that $v_{H H}^{1} \square y$. Straightforward calculations (presented in the Appendix) establish that this condition can be satisfied when firms are sufficiently patient:

Proposition 2. There exists a $\delta^{\text {lin }}<1$ such that, for all $\delta>\delta^{\text {lin }}$, there exist values $y>$ $x>0$ such that the line segment $[(x, y),(y, x)]$ is in the set of informative PPE values, $V^{I}$. Each utility pair $\mathbf{u}$ on the segment can be implemented using a policy vector $(\mathbf{p}, \mathbf{q}, \mathbf{v})$ such that pricing efficiency holds, $v_{j k} \in[(x, y),(y, x)]$ for each $(j, k)$, and $q_{L H}^{1}+q_{H L}^{2}=1+\frac{\delta\left(\square+\eta_{L}^{2}\right)}{1+\square-\delta \eta_{H}}$, where $\square=\left(r-\theta_{H}\right) /\left(\theta_{H}-\theta_{L}\right)$.

For the parameter values we use in our computations $\left(r=2.5, \theta_{H}=2, \theta_{L}=1\right.$ and $\left.\eta_{L}=.6\right), \delta^{l i n} \approx .7$, and at that discount factor, $q_{L H}^{1}+q_{H L}^{2} \approx 1.5$, less than the first-best value of 2 . In fact, it is straightforward to verify that $q_{L H}^{1}+q_{H L}^{2}<1+\delta$, and so this scheme never yields first-best profits, even when $\delta=1$; this is because we have imposed the condition that IC-Off $1_{L H}^{I}$ is binding when implementing $(x, y)$. When $\delta$ gets larger, this restriction is too strong; a more profitable linear self-generating region exists where only $\mathrm{IC}_{\mathrm{O}} \mathrm{Off} \mathrm{I}_{L L}^{I}$ binds when implementing the endpoints. Although the Pareto superior sets can be computed as well, we do not pursue it here.

\subsection{The Shape of the Pareto Frontier}

We proceed now to consider the shape of the Pareto frontier of the informative PPE utility set. As discussed at the start of Section 4, when firms attempt to implement highly asymmetric equilibrium values, the off-schedule incentive constraints bind and some inefficiency may be required. We thus anticipate that total cartel profits fall as values become more asymmetric, indicating that the frontier is typically nonlinear. In the present subsection, we establish conditions under which a subset of the Pareto frontier of the set of informative 
PPE values is a line with slope equal to -1 . In addition, we formally characterize the manner in which the off-schedule constraints determine the boundaries of this linear subset (as well as the boundaries of the frontier itself).

To begin, we recall our assumption that firms can randomize between continuation equilibria, which ensures that firms have available a convex set of continuation values at any point in time. Figure 2 illustrates the general shape of a symmetric, convex set of continuation values. The set has four "corners," labelled as North, South, East, and West, or $v_{N}, v_{S}, v_{E}, v_{W}$, where $v_{N}=\left(v_{N}^{1}, v_{N}^{2}\right)$ and likewise for the other corners. Between two corners, the boundary of the set is monotone. The part of the boundary between $v_{N}$ and $v_{E}$ is of particular interest to us, since it represents the Pareto frontier of the set of continuation values. In this context, we say that a scheme is characterized by continuation value Pareto inefficiency if for some $(j, k),\left(v_{j k}^{1}, v_{j k}^{2}\right)$ is not on the Pareto frontier of $V$.

When describing the Pareto frontier of the set of feasible continuation values given a set $V$, we use the notation

$$
f\left(v_{j k}^{1}\right)=\left\{\begin{array}{cc}
\max \left\{v_{j k}^{2}:\left(v_{j k}^{1}, v_{j k}^{2}\right) \in c o(V)\right\} & \text { if } v_{j k}^{1} \in\left[v_{N}^{1}, v_{E}^{1}\right] \\
v_{N}^{2} & \text { if } v_{j k}^{1}<v_{N}^{1} \\
-C \cdot\left(v_{j k}^{1}-v_{E}^{1}\right) & \text { if } v_{j k}^{1}>v_{E}^{1}
\end{array}\right.
$$

for some large constant $C$. Of course, convexity of the set $V$ implies concavity of the frontier $f$. We define the function $f$ outside the domain of the Pareto frontier in order to simplify the statement of some of our results about the slope of the frontier.

Given our assumption that firms are symmetric, $f(v)+v$ is maximized at $v^{1}=v_{s}^{1}$, where $f\left(v_{s}^{1}\right)=v_{s}^{1}$. We may thus say that a scheme is characterized by future inefficiency if $v_{j k}^{1}+f\left(v_{j k}^{1}\right)<2 v_{s}^{1}$ for some $(j, k)$, so that under some state the continuation values fail to maximize total cartel future profits. As mentioned above, future inefficiencies are associated with highly asymmetric values, and represent an efficiency cost that is incurred when firms attempt to provide incentives with such values. Thus, it is important to identify conditions under which a subset of the Pareto frontier has slope of -1 , so that the firms may make some use of future market-share favors without efficiency loss.

For the informative PPE set $V^{I}$, let $v_{s}^{I}$ be the point on the Pareto frontier of $V^{I}$ that provides equal utility to both firms. Consider a policy vector that implements $v_{s}^{I}$, and assume that the off-schedule constraints do not bind in states $(L, L)$ and $(H, H)$. Suppose for simplicity that pricing efficiency is used. By lowering firm 1's market share in state $(L, L)$ by $\varepsilon / \eta_{L}$ and $(H, H)$ by $\varepsilon / \eta_{H}$, it is possible to transfer market share from firm 1 to firm 2 without upsetting any of the on-schedule incentive constraints. This new scheme is also feasible. While firm 1's profit is lower, total cartel profit is unchanged, and so the Pareto frontier has an interval with slope equal to -1 .

How can we ensure that the off-schedule constraints do not bind in states $(L, L)$ and $(H, H)$ ? Without loss of generality, when implementing $v_{s}^{I}$, we may specify that $q_{j j}^{1}=1 / 2$ 
and $v_{j j}=v_{s}^{I}$ for $j \in\{L, H\}$. With this specification in place, and observing that the most demanding circumstance from the perspective of off-schedule constraints arises in state $(L, L)$ when $p_{L L}=r$, we see that it suffices to check the following condition:

$$
\left(r-\theta_{L}\right) /(2 \delta)<v_{s}^{1 I}-\underline{v}^{1} .
$$

Of course, $v_{s}^{1 I}$ is endogenously determined. We illustrate a range of discount factors where (OffSS) holds in our computational examples. Further, Proposition 2 establishes a lower bound for $v_{s}^{1 I}$ : if we define $x$ and $y$ as in Section 4.2, since $[(x, y),(y, x)]$ is contained in $V^{I}$, $v_{s}^{1 I}$ must be greater than $(x+y) / 2$. Using straightforward computations, it can be shown that if $\left(r-\theta_{H}\right) /\left(\theta_{H}-\theta_{L}\right)>.275,^{28}$ then (OffSS) holds for all $\delta>\delta^{l i n}$. In other words, (OffSS) holds whenever the linear self-generating set constructed in Section 4.2 exists.

When (OffSS) holds, we have the following initial characterization of the shape of the Pareto frontier:

Proposition 3. Assume (OffSS).

(i) The Pareto frontier of $V^{I}$ has an open interval with slope equal to -1.

(ii) Let $(x, y)$ and $(y, x)$ denote the endpoints of this open interval, and let $(\mathbf{p}, \mathbf{q}, \mathbf{v})$ implement $(x, y)$. Then at least one of the following two conditions hold: (a) for some $j \in\{L, H\}$, IC-OffI $I_{j j}^{I}$ binds; (b) $v_{j j}^{1} \square x$ for some $j \in\{L, H\}$, and either $p_{j j} \square \theta_{j}$ or $q_{j j}^{1}=0$ for some $j \in\{L, H\}$.

Part (i) confirms the existence of a subset of the Pareto frontier with slope -1 . Part (ii) then identifies the factors that limit this subset. Formally, either an off-schedule constraint binds, or else the firms run out of market-share favors and the ability to shift continuation values in the event of ties. In either case, the firms cannot implement any further transfer of utility away from firm one and towards firm two without a loss of efficiency. For firms of moderate patience $\left(\delta<\delta^{F B}\right)$, the off-schedule constraints typically bind first. It is possible to further characterize the implementation of $(x, y)$ for different parameter regions, but we will not pursue it here.

Next, we observe that the off-schedule constraints also determine the endpoints of the entire Pareto frontier and that they force the firms to bear inefficiency when implementing those endpoints.

Proposition 4. Suppose that $(\mathbf{p}, \mathbf{q}, \mathbf{v})$ implements $v_{N}^{I}$. (i) If IC-Oni $i_{U}$ is slack for each $i$, then there is either pricing inefficiency, productive inefficiency or both. (ii) If $\bar{q}_{L}^{i}>\bar{q}_{H}^{i}$ for each firm $i$, at least one of the following two conditions hold: (a) for some $j \in\{L, H\}$, IC-Off $1_{j j}^{I}$ binds; (b) $v_{j j}^{1} \square v_{N}^{1 I}$ for some $j \in\{L, H\}$, and either $p_{j j} \square \theta_{j}$ or $q_{j j}^{1}=0$ for some $j \in\{L, H\}$.

\footnotetext{
${ }^{28}$ The lower bound on $\left(r-\theta_{H}\right) /\left(\theta_{H}-\theta_{L}\right)$ is calculated when $\eta_{L}=1 / 2$. This bound is decreasing in $\eta_{L}$, and so the bound is relaxed when $\eta_{L}>1 / 2$.
} 
To understand part (i), observe that if the firms are using productive and pricing efficiency, then IC-Off $1_{L H}^{I}$ and IC-Off-M1 are slack. So long as the upward on-schedule incentive constraints are slack (as they are in all of our computational examples), ${ }^{29}$ firm 1 can given up some market share in state $(L, H)$ without violating any incentive constraints. In other words, the off-schedule constraints create a force in favor of sacrificing productive efficiency when firms attempt to implement asymmetric continuation values. Part (ii) is similar to Proposition 3 (ii). If neither of the off-schedule constraints bind, it should be possible to shift market shares or continuation values in the event of ties from firm 1 to firm 2. In contrast to Proposition 3 (ii), however, shifting continuation values may entail an efficiency loss; indeed, the frontier will typically have slope greater than -1 near the corner, $v_{N}^{I}$.

Finally, we consider whether the set of equilibrium values is itself convex. Since payoffs and constraints are nonlinear in market shares and prices (they depend on $q_{j k}^{1}, q_{j k}^{1} \cdot p_{j k}$ and $\left.\left(1-q_{j k}^{1}\right) \cdot p_{j k}\right), \mathcal{F}^{I}(V)$ is not generally convex, and $V^{I}$ may not be convex either. When prices are the same in two distinct equilibria, however, the nonlinearity does not pose a problem, and the convex combination of two equilibrium values can be implemented using a convex combination of the two associated policy vectors. In the next subsection, we analyze conditions under which prices are always equal to the reservation value $r$ when implementing values on the Pareto frontier of the equilibrium set.

\subsection{Pricing and Continuation Value Efficiency}

In this section, we consider whether, when implementing Pareto efficient informative PPE values, pricing and continuation-value Pareto efficiency are used. We identify two distinct circumstances under which we expect these properties to hold. We then show that these properties imply that the downward on-schedule constraints are typically binding.

To begin, we consider whether pricing and continuation-value Pareto efficiency hold when implementing equilibrium values such that: (i) the off-schedule constraints are slack, and (ii) the Pareto frontier is sufficiently wide, and the equilibrium value is sufficiently far from the corners of the Pareto frontier, $v_{N}^{I}$ and $v_{E}^{I}$, that the firms implement the equilibrium value using continuation values strictly between $v_{N}^{I}$ and $v_{E}^{I}$. As Proposition 1 indicates, these properties hold when implementing values in the neighborhood of $v_{s}^{I}$ for discount factors that exceed $\delta^{F B}$. However, conditions (i) and (ii) apply in a wider set

\footnotetext{
29 To further understand the role of the assumption that IC-Oni $i_{U}$ is slack, first observe that if both downward and upward on-schedule constraints bind for firm $i$, then $\vec{q}_{L}^{i}=\vec{q}_{H}^{i}$, which requires productive incfficiency. Part (ii) docs not cover the case where IC-Oni $i_{U}$ binds but IC-Oni $i_{D}$ is slack for one or both firms. Although we do not formally analyze this case, we establish below conditions under which the upward on-schedule constraints are slack at the start of the game, when implementing $v_{s}^{I}$; adjusting the scheme so that the upward on-schedule constraints bind typically requires new inefficiencies, consistent with the proposition.
} 
of circumstances. In particular, for a range of more moderate discount factors, the offschedule constraints are slack at the begi

) implement a Pareto efficient utility pair in $V^{I}$. Suppose that the off-schedule constraints hold with slack, and $v_{N}^{1 I}<v_{j k}^{1}<v_{E}^{1 I}$ for all $(j$,

that firms use Pareto

efficient continuation values. In establishing this result, we must examine two possibilities. Suppose first that there exists a cost state $j$ for firm 1 such that firm 2's continuation value is always (i.e., in both states $(j, L)$ and $(j, H))$ below the frontier. In this case, we can simply add a constant to firm 2's continuation value whenever firm 1 experiences cost state $j$. This raises firm 2's utility without disturbing its incentive constraints. The second possibility is more subtle. Suppose that there exists a cost state $j$ for firm 1 such that firm 2's continuation value is below the frontier in exactly one cost state for firm 2. For example, we might have $v_{j H}^{2}=f\left(v_{j H}^{1}\right)$ and $v_{j L}^{2}<f\left(v_{j L}^{1}\right)$. In this event, we may reduce $v_{j H}^{1}$ and increase $v_{j L}^{1}$ (at rates $\eta_{L}$ and $\eta_{H}$, respectively), while holding the continuation values for firm 2 constant, without disturbing firm 1's ex ante utility or incentive constraints. Provided that the initial continuation values lie strictly between the corners of the Pareto frontier, this maneuver positions firm 2's continuation values, in states $(j, H)$ as well as $(j, L)$, strictly below the frontier. But we may now apply the argument developed for our first possibility, adding a constant to firm 2's continuation value whenever firm 1 experiences cost state $j$, and thereby increase firm 2's utility.

Part (ii) establishes the optimality of efficient pricing. The result follows because it is always possible to raise prices and lower continuation values to keep the firms indifferent; but we have just established that continuation values below the frontier can be strictly improved upon.

Our next objective is to relax the constraint that the continuation values lie strictly between the corners of the Pareto frontier. We find that additional restrictions on parameters are required to maintain our efficiency results in this case. In a computational example, Figure 5 illustrates our approximation of the Pareto frontier for a set of parameters such that, at the start of the game, when the firms attempt to implement equal utility, the off-schedule constraints do not bind (in particular, the off-schedule constraints are slack for 
the $(\mathbf{p}, \mathbf{q}, \mathbf{v})$ implementing states 7-17). However, the Pareto frontier is not wide enough to implement full productive efficiency using Pareto-efficient transfers (since the off-schedule constraints do bind for the "corner" continuation values, that is, at the edges of the Pareto frontier).

Proposition 6. Suppose that (Pool) holds. Suppose that (p, q, v) implements a Pareto efficient utility pair in $V^{I}$, the off-schedule constraints hold with slack, and further either (a) both $v_{H H}$ and $v_{L L}$ are on the interior of the line segment on the Pareto frontier of co $(V)$ with slope equal to -1 , or (b) $0<q_{j j}^{1}<1$ for $j \in\{L, H\}$. Then: (i) continuation values are Pareto efficient, and (ii) prices are efficient.

This result generalizes Proposition 5, under several additional restrictions. To understand the restrictions, recall Lemma 5, which establishes that when (Pool) holds, firms increase the total collusive profit by decreasing productive efficiency and increasing the efficiency of prices and continuation values. ${ }^{30}$ As an extension of Lemma 5 , this result implies that when the continuation value frontier is narrow, and firms maximize the sum of cartel profits, they choose productive inefficiency over inefficient prices and continuation values.

Continuing with the case in which the off-schedule incentive constraints are slack, a natural question is whether the Pareto frontier is itself self-generating. Are there circumstances in which the implementation of a Pareto efficient equilibrium utility pair requires for some state a Pareto inefficient continuation value (i.e., a "price war")? If such a circumstance were to arise, then it would be associated with an attempt to implement an asymmetric utility pair, with the continuation-value inefficiency then employed as a means to transfer utility between agents. Furthermore, as Proposition 6 Part (i) indicates, for an efficient utility pair, Pareto inefficient continuation values would b

, however, it is conceivable that an optimal cartel might implement a utility transfer with an inefficient continuation value, particularly in the $(L, L)$ state, so as to draw utility from a firm while simultaneously relaxing that firm's downward on-schedule incentive constraint. We note, though, that this possibility does not arise in any of our computational examples.

So far, we have considered only the on-schedule constraints. Before proceeding, we make two observations about the effects of off-schedule constraints on pricing and continuation-

\footnotetext{
${ }^{30}$ Although we do not formalize this, it can be shown that when (Pool) fails, the sum of firm profits is maximized using productive efficiency, even at the expense of $i$

plementing asymmetric utility vectors, the firms may use both pricing and continuation value inefficiency. We will not pursue this case further here.
} 
value efficiency. First, the off-schedule constraints place downward pressure on the prices. The off-schedule constraints may require lower prices for low-cost firms, in order to reduce the incentive that such firms have to undercut the equilibrium price. However, as lowering prices reduces total cartel profits, lowering prices does not necessarily help firms achieve Pareto efficient equilibrium values. When prices are inefficient, in principle, the upward onschedule constraints might then bind, although we have not discovered any examples where this occurs. Second, in establishing continuation-value Pareto efficiency, we employed arguments in which firms shift profits across states of the world. When off-schedule constraints bind, the profit of a firm in a particular state of the world may be constrained. Thus, our characterizations are more limited. However, in the next subsection, we establish a set of characterizations of productive efficiency that hold even in the presence of off-schedule constraints.

Returning to the case where the off-schedule constraints are slack, we now examine the consequences of pricing and continuation-value Pareto efficiency. In particular, we establish that the downward on-schedule constraints then bind, unless the firms are indifferent among a range of equally-desirable implementation schemes (as might occur if the continuation values lie on a linear segment of the frontier).

Proposition 7. Let $\mathbf{u}$ be a Pareto efficient utility pair in $V^{I}$. Whenever there exists a policy vector implementing $\mathbf{u}$ such that prices are efficient and continuation-value Pareto efficiency holds, then there exists a $(\mathbf{p}, \mathbf{q}, \mathbf{v})$ that implements $\mathbf{u}$ such that either: (a) at least one off-schedule constraint is binding, or (b) (1) IC-Oni $i_{D}$ binds for each $i$, and (2) if $\bar{q}_{L}^{i}>\bar{q}_{H}^{i}$ for each $i$, then $v_{L H}^{1} \square v_{j j}^{1} \square v_{H L}^{1}$ for each $j \in\{L, H\}$.

This result explores the consequences of concavity of the frontier, $f(v)$. Concavity implies that asymmetric continuation values are associated with future inefficiency. Thus, unless behavior is constrained by off-schedule considerations, it is only optimal to use asymmetric continuation values to the extent that they provide a mechanism to enhance productive efficiency benefits. If the frontier is strictly concave in the relevant region, the firms use asymmetric continuation values up to the point that the downward IC-On constraints bind. To see this, suppose that $v_{H L}^{1}>v_{L L}^{1}$ and IC-On $1_{D}$ is slack. Then, the incentives are stronger than necessary: we can bring $v_{H L}^{2}$ and $v_{L L}^{2}$ toward one another at exactly the rates that leave firm 2's on-schedule incentives undisturbed $\left(\eta_{L}\right.$ and $\eta_{H}$, respectively). If we simultaneously move firm 1's continuation values along the Pareto frontier, this adjustment will not disturb firm 1's on-schedule incentives because IC-On $i_{D}$ is slack. Such an adjustment leaves $\bar{U}^{2}$ unchanged, and increases $\bar{U}^{1}$ whenever the frontier is strictly concave. In contrast, if the frontier is linear in the relevant region, the firms will be indifferent to the adjustment. In any case, only the off-schedule constraints would prevent such an adjustment. 
Finally, observe that the ranking of continuation values in (b)(2) conforms with the intuition that firm 1 is rewarded in state $(H, L)$ and punished in state $(L, H)$, in order to deter a (downward) on-schedule deviation. The additional assumption that $\bar{q}_{L}^{i}>\bar{q}_{H}^{i}$ for each $i$ is included because under that assumption, the upward and downward on-schedule incentive constraints cannot bind at the same time for a given firm. Since (b)(1) specifies that the downward on-schedule constraints bind, the assumption implies that the upward on-schedule incentive constraints are slack. ${ }^{31}$

\subsubsection{Computational Examples}

Consider Figure 4 in relation to Proposition 5. As illustrated in the diagram, neither the off-schedule constraints nor the constraints on the width of the Pareto frontier are binding for the policy vectors that implement states 11 to 20 , and thus our characterizations from Proposition 5 apply for those states. The table of transitions illustrates that continuationvalue Pareto efficiency holds: in every state, after every realization of cost types, the firms move to another state on the Pareto frontier. Given that this property holds, the diagram only labels and represents the Pareto frontier. The continuation values are ranked as predicted by Proposition 7: for firm 1, the realization $(L, H)$ is followed by a low continuation value, while the realization $(H, L)$ is followed by a high continuation value. Similar results hold in the example illustrated in Figure 5. In this case, the conditions of Proposition 6 are satisfied when implementing states 9-17.

\subsection{Productive Efficiency}

Having considered the use of pricing and continuation-value efficiency, we next explore whether firms use productive efficiency when implementing Pareto efficient utilities.

Our first result applies to all points on the Pareto frontier, with no additional restrictions. It hinges upon the tradeoff between productive efficiency today and future inefficiency tomorrow. To see the logic, suppose $q_{L H}^{1}<1$, and consider whether firms could engineer a Pareto superior equilibrium. The firms could increase productive efficiency today by increasing $q_{L H}^{1}$. To maintain on-schedule incentive compatibility for firm $1, v_{L H}^{1}$ could then be reduced so that the combined adjustments in $q_{L H}^{1}$ and $v_{L H}^{1}$ do not increase in $U^{1}(L, H ; \mathbf{z})$. Since a firm values an increase in market share less when its costs are high, this is assured if the adjustments are made so as to leave $U^{1}(L, L ; \mathbf{z})$ unchanged. Suppose this adjustment is made, and now consider firm 2. This firm experiences a reduction in $q_{L H}^{2}$ and, moving along the frontier, an increase in $v_{L H}^{2}$. As market share is more valuable when costs are low, the positive effect of the increase in $q_{L H}^{1}$ on $U^{1}(L, L ; \mathbf{z})$ is greater than the

\footnotetext{
${ }^{31}$ If $\bar{q}_{L}^{i}=\bar{q}_{H}^{i}$ for some $i$, the rankings of continuation values are not quite as sharp, and we do not formally delineate them.
} 
complementary negative effect of the decrease in $q_{L H}^{2}$ on $U^{2}(H, H ; \mathbf{z})$. The continuationvalue reduction that leaves $U^{1}(L, L ; \mathbf{z})$ unchanged is therefore sufficient to strictly increase $U^{2}(H, H ; \mathbf{z})$, if firm 1's continuation-value reduction were to translate, dollar for dollar, into a continuation-value gain for firm 2 . Indeed, even if the frontier is strictly concave, if the future inefficiency is not too great (i.e., if the frontier is not too flat), then this maneuver raises $U^{2}(H, H ; \mathbf{z})$. A Pareto superior equilibrium is thus generated, provided that the new arrangement continues to satisfy the off-schedule incentive constraints. ${ }^{32}$ But this, too, is assured, since from the perspective of the off-schedule constraints, it does not matter whether market share or continuation values are used to provide profits in a given state (in contrast, higher prices raise the incentive to deviate).

Formally, we have the following proposition:

Proposition 8. Choose any Pareto efficient utility pair in $V^{I}$ and let $(\mathbf{p}, \mathbf{q}, \mathbf{v})$ be the policy vector that implements this pair. Then productive efficiency holds in state $(L, H)$ (i.e., $q_{L H}^{1}=1$ ) if $p_{L H}>\theta_{H}$ and if there exists $\varepsilon>0$ such that

$$
1+\Delta_{\varepsilon}^{-} f\left(v_{L H}^{1}\right) \equiv 1+\left[f\left(v_{L H}^{1}\right)-f\left(v_{L H}^{1}-\varepsilon\right)\right] / \varepsilon<\left(\theta_{H}-\theta_{L}\right) /\left(p_{L H}-\theta_{L}\right) .
$$

Productive efficiency holds in state $(H, L)$ (i.e., $\left.q_{H L}^{1}=0\right)$ if $p_{H L}>\theta_{H}$ and if there exists $\varepsilon>0$ such that

$$
1+\Delta_{\varepsilon}^{+} f\left(v_{H L}^{1}\right) \equiv 1+\left[f\left(v_{H L}^{1}+\varepsilon\right)-f\left(v_{H L}^{1}\right)\right] / \varepsilon>-\left(\theta_{H}-\theta_{L}\right) /\left(p_{H L}-\theta_{L}\right) .
$$

Consistent with the description above, we see that productive efficiency is used when implementing Pareto efficient utilities, if the continuation values in the $(L, H)$ and $(H, L)$ states are drawn from regions of the frontier at which the frontier slope does not depart too greatly from -1 .

Recall that by Proposition 3, when (OffSS) holds, the interior of the Pareto frontier has slope equal to -1 . Thus, Proposition 8 can be used to show that, when (OffSS) holds, some productive efficiency is used by optimally colluding firms at the start of the game. To see why, observe that when (OffSS) holds, it is feasible to implement a symmetric scheme with no productive efficiency using pricing efficiency and $v_{j k}=v_{s}^{I}$ for all $(j, k)$; clearly, this is the best scheme with no productive efficiency. However, Proposition 8 implies that the latter scheme is Pareto dominated by a scheme with at least some productive efficiency, implemented using $v_{L H}$ and $v_{H L}$ (at least weakly) outside the interval of the Pareto frontier with slope -1 .

It is important to emphasize, though, that Proposition 8 provides sufficient, but not necess 
constraints are slack (as when the conditions of Proposition 7 hold), and utility is transferrable without efficiency loss, as in Proposition $6 .^{33}$ However, in order to state necessary and sufficient conditions for productive efficiency, we must confront the possibility that a range of policy vectors implements the same equilibrium values. In such cases, we focus on equilibria that satisfy two criteria. First, we select the policy vector with the highest level of productive efficiency. Second, we focus on schemes that are symmetric outside of states where the cost types are equal; that is, the schemes should treat firm 1 in state $(L, H)$ the same as firm 2 in state $(H, L)$. To see that such a scheme exists, observe that as long as utility is transferrable, the optimal scheme maximizes the sum of firm profits, and transfers utility in states $(L, L)$ and $(H, H)$; the symmetry of the model then implies the desired symmetry of policy vectors.

Proposition 9. Suppose that $(\mathbf{p}, \mathbf{q}, \mathbf{v})$ implements an equilibrium value $\mathbf{u}$ on the Pareto frontier of $V^{I}$ and either assumption (a) or (b) of Proposition 6 is satisfied. Further, suppose that the off-schedule constraints and $I C$-Oni $i_{U}$ are slack for each $i$, that $p_{L H}=p_{H L}>\theta_{H}$, $q_{L H}^{1}=q_{H L}^{2}, v_{L H}^{1}=v_{H L}^{2}$, and that no other such policy vector implements u using a larger $q_{L H}^{1}$. Then $q_{L H}^{1} \in(0,1)$ and $q_{H L}^{1} \in(0,1)$ if and only if for all $\varepsilon>0$,

$$
1+\Delta_{\varepsilon}^{+} f\left(v_{L H}^{1}\right) \square \frac{\theta_{H}-\theta_{L}}{p_{L H}-\theta_{H}+\eta_{L}\left(\theta_{H}-\theta_{L}\right)}<1+\Delta_{\varepsilon}^{-} f\left(v_{L H}^{1}\right) .
$$

Further, $q_{L H}^{1}=1 / 2$ and $q_{H L}^{1}=1 / 2$ if and only if the second inequality holds at $v_{H L}^{1}=$ $v_{L H}^{1}=v_{s}^{I 1}$, while $q_{L H}^{1}=1$ and $q_{H L}^{1}=0$ if and only if the second inequality fails.

One implication of this result is that if $v_{E}^{1 I}-v_{N}^{1 I}<\left(r-\theta_{H}\right) / \delta$ (the minimum width required to implement productive efficiency using pricing and continuation-value Pareto efficiency), there is productive inefficiency even in the first period of play, when implementing $v_{s}^{I}$, so long as the off-schedule constraints do not bind. Further, we see that if future inefficiency is extreme, so that $\Delta_{\varepsilon}^{-} f\left(v_{s}^{I 1}\right)=0$, the second inequality in (4.4) holds when $p_{L H}=r$ if and only if (Pool) holds. Thus, we can interpret Proposition 9 as a generalization of Lemma 5. In general, firms implement some productive efficiency; however, they stop short of full productive efficiency if the slope of the frontier gets too steep or too flat, and in particular, if the frontier is too narrow. ${ }^{34}$

${ }^{33}$ The approach of Proposition 9 can be extended to the case where utility is not transferrable across firms; however, the conditions are more cumbersome and more stringent.

${ }^{34}$ Additional characterizations can be provided. For example, if the upward on-schedule constraints are slack, then for $\varepsilon$ sufficiently small, $\Delta_{\varepsilon}^{-} f\left(v_{H H}^{1}\right)<-1$, then $q_{H H}^{1}=1$, and if $\Delta_{\varepsilon}^{+} f\left(v_{H H}^{1}\right)>-1$, then $q_{H H}^{1}=0$. In other words, the firms take the market shares to the extreme before incurring future inefficiency in state $(H, H)$. It is also possible to show that, when off-schedule constraints are slack, $f$ is differentiable, and all continuation values are interior, Pareto efficient points require $f^{\prime}\left(v_{L H}^{1}\right) \cdot f^{\prime}\left(v_{H L}^{1}\right)=f^{\prime}\left(v_{L L}^{1}\right) \cdot f^{\prime}\left(v_{H H}^{1}\right)$. Intuitively, the continuation values are chosen to balance the inefficiencies incurred in each state of the world. 
Let us now summarize our characterizations of Pareto efficient collusive schemes for firms of moderate patience. First, we find that firms are willing to bear some future inefficiency to gain productive efficiency in the present. However, when off-schedule constraints do not bind, and either the Pareto frontier is wide enough or (Pool) holds, the firms maintain pricing and continuation-value Pareto efficiency, even at the expense of productive efficiency. In contrast, the firms may sacrifice pricing and continuation-value Pareto efficiency when they attempt to implement asymmetric equilibrium values, if the off-schedule incentive constraints directly or indirectly prevent the use of future market-share favors in the event of ties. Thus, for firms of moderate patience, we expect to start the game using fairly efficient schemes (at worst, there is some productive inefficiency), but the schemes may incorporate additional inefficiencies following a series of one or more cost realizations whereby one firm has lower cost than another.

\subsubsection{Computational Examples}

Consider again Figure 4. Notice that for a wide range of states (9 to 22), (approximately) productive efficiency is implemented, as predicted by Proposition 8: at the extreme continuation values (in states $(L, H)$ and $(H, L))$ associated with these states, the slope of the frontier is always within the bounds specified in (4.2) and (4.3). Further, states 9 to 21 use the same extreme continuation values $\left(v_{L H}=24\right.$ and $\left.v_{H L}=3\right)$; these are labeled on the diagram. Due to concavity of the frontier, shifting these continuation values to become asymmetric would lead to greater future inefficiency. Thus, in order to implement a range of equilibrium values, firms find it optimal to hold the extreme continuation values fixed, and shift the market shares and continuation values in ties. Naturally, there is no efficiency loss from shifting market shares in the event of ties; likewise, since the inter the frontier is linear (as predicted by Proposition 3), there is no efficiency loss in shifting continuation values along the linear portion of the frontier.

Further, we observe that the firms use productive inefficiency when implementing the extreme values of the Pareto frontier. In particular, states 3 and 24 use productive inefficiency; since these states (or less efficient ones) are reached with positive probability from every starting point, even the most profitable points on the Pareto frontier yield less than the first-best profits. This is consistent with Proposition 8: when implementing state 24, $v_{L H}=26$, the corner of the Pareto frontier. Thus, (4.2) fails, so that productive efficiency is not necessarily optimal.

Now consider Figure 5. Across all but the most extreme states, the overall level of productive efficiency is approximately the same, with $q_{L H}^{1}+q_{H L}^{2}$ approximately equal to 1.57 , and incentives for truth-telling are provided in a similar fashion, with the firms going to state 1 following a realization of $(H, L)$ and to state 20 following a realization of $(L, H)$. These states correspond to the corners of the frontier, and so the fact that firms achieve 
only partial productive efficiency is consistent with Proposition 8. Along the interior of the frontier, the slope of the frontier stays fairly close to -1 , and this indicates that the firms are willing to incur some future inefficiency in order to implement partial productive efficiency. ${ }^{35}$ However, consistent with Proposition 6, the firms do not use continuation-value inefficiency to implement higher levels of productive efficiency.

These features imply that the interior of the frontier is nearly linear. However, to implement the extreme states, somewhat greater productive inefficiency is required. Thus, optimally colluding firms capture some productive efficiency in the first period of the game, but at the cost of greater inefficiency in the future. ${ }^{36}$

Outside of states 7-17, the off-schedule constraints bind, and at the extreme points of the frontier we have computed, it is impossible to decrease the utility of the disadvantaged firm any further while maintaining pricing efficiency. As our computational algorithm does not allow for pricing inefficiency, we have not determined if the firms could do better by lowering prices. ${ }^{37}$

\section{Informative v. Uninformative Communication}

We now consider the role of communication. We begin by contrasting the case of informative communication with the opposite possibility, where firms are unable or unwilling to communicate. Recall that Section 2 specifies a particular extensive-form game in which communication occurs first, and then firms make pricing decisions and market-share proposals. However, market-share proposals only affect outcomes in the event that prices are equal. The main insights of Section 4 are robust to alternative specifications of the extensive-form game, so long as the firms have an instrument to divide the market on a state-contingent basis, and so long as pricing decisions are taken after communication takes place. However, when we consider how the game progresses in the absence of communication, the timing assumptions play a much more important role.

To begin, let us take the specific extensive-form game of Section 2. Interpreted literally, the absence of communication implies that the firms use the announcement $\alpha^{i}\left(\theta^{i}\right)=N$

${ }^{35}$ When implementing states 1 and $2, q_{L H}^{1} \approx 1$ but the overall level of productive efficiency is lower; thus, the firms go to state 18 or 19 following state $(L, H)$ rather than state 20 .

${ }^{36}$ Relative to the linear self-generating set constructed in Section 4.2, however, the level of productive efficiency is higher (1.57 in the computation versus 1.5 for the strictly linear set). Intuitively, within the linear set, the level of productive efficiency is the same when implementing each point in the set. In contrast, in Figure 5, states 1, 2, and 20 are available only because the firms sacrifice productive efficiency in those states. With those (less efficient) equilibrium values in the set, firms have greater future market share favors available, and thus greater productive efficiency is attained at the start of the game.

${ }^{37}$ However, since the advantaged firm produces at least half the time in each state, lowering the price in any state would apparently lower profits for both firms, and thus leave the Pareto frontier. But, a more rigorous argument is required to establish such a result, since we must consider more radical adjustments in conjunction with a price decrease, such as reallocating market shares across states of the world. 
(which we will now suppress in our notation) in all states of the world. Now consider market-share proposals. It might be argued that market-share proposals are inherently linked to communication; we could formally accommodate such an alternative assumption by requiring that following the no-communication announcement $N, \varphi^{1}\left(\theta_{j}\right)=\varphi^{2}\left(\theta_{k}\right)=1 / 2$ for all $j, k$. Although the main qualitative ideas described in this section apply when we make the latter assumption, for consistency we instead follow explicitly the model of Section 2 , whereby equally-priced firms are able to unilaterally withhold quantity from the market even in the absence of explicit communication. The difference is that a firm's price and market-share proposals must then be decentralized, as these variables depend only on the firm's own cost type.

In this context, how are the firms affected by requiring announcements to be uninformative? This requirement has both a downside and an upside. The downside is that the set of market-sharing arrangements that can be implemented is restricted. This restriction, however, is perhaps less severe than might be expected: in our model, even without informative communication, market share still can be allocated in a state-contingent fashion, provided that the allocation is consistent with decentralized decision-making. ${ }^{38}$ For example, the simplest no-communication scheme might require $\rho^{2}\left(\theta_{H}\right)=\rho^{1}\left(\theta_{H}\right)>\rho^{2}\left(\theta_{L}\right)=\rho^{1}\left(\theta_{L}\right)$, and $\varphi^{1}\left(\theta_{j}\right)=\varphi^{2}\left(\theta_{k}\right)=1 / 2$ for all $j, k$ : this yields productive efficiency, equal market shares in the event of ties and even pricing efficiency (in our Bertrand setting, arbitrarily small price differences can allocate consumers). Similarly, by setting $\rho^{2}\left(\theta_{H}\right)>\rho^{1}\left(\theta_{H}\right)>\rho^{2}\left(\theta_{L}\right)>$ $\rho^{1}\left(\theta_{L}\right)$, the firms may continue to achieve productive and pricing efficiency, but now firm 1 wins all ties.

Notice that the two schemes just described could be implemented even if we eliminated the market-share proposals altogether, if we instead maintained the assumption that consumers divide themselves evenly among two firms charging the same price. However, when decentralized quantity restrictions are included, the firms might specify a scheme whereby $\rho^{2}\left(\theta_{H}\right)=\rho^{1}\left(\theta_{H}\right)>\rho^{2}\left(\theta_{L}\right)=\rho^{1}\left(\theta_{L}\right)$, and $\varphi^{1}\left(\theta_{j}\right)=2 / 3, \varphi^{2}\left(\theta_{k}\right)=1 / 3$. This allows the firms to share the market unequally in the event of ties. They could also use a scheme whereby $\rho^{2}\left(\theta_{H}\right)=\rho^{2}\left(\theta_{L}\right)=\rho^{1}\left(\theta_{H}\right)>\rho^{1}\left(\theta_{L}\right)$, and $\varphi^{1}\left(\theta_{j}\right)=2 / 3, \varphi^{2}\left(\theta_{k}\right)=1 / 3$. This scheme allows firm 1 to serve the market in its entirety in state $(L, H)$, while firm 1 receives $2 / 3$ of the market in other states. Despite these possibilities, many market-sharing arrangements are infeasible without informative communication. ${ }^{39}$

\footnotetext{
${ }^{38}$ Compare our model to a Cournot model, where without communication, colluding firms typically suffer large inefficiencies: a high-cost firm produces, just in case the opponent is high-cost as well, but this is wasteful if the opponent turns out to be low cost. In contrast, when the firms can communicate, they are able to select the monopoly output for the cost type of the firm that actually produces, and other firms refrain from producing. Thus, our base model presents a conservative case for communication.

${ }^{39}$ This discussion highlights the role of our assumption that the game has simultaneous moves: the firms might wish to move sequentially, if that were possible, to restore the ability to assign market shares in a fully state-contingent manner.
} 
An environment without informative communication also admits an upside for the colluding firms, once the off-schedule constraints are considered. In the absence of informative communication, each firm must be dissuaded from deviating from the collusive agreement after observing its own type, but before knowing the type of the other firm. In other words, the off-schedule incentive constraints bind at the interim stage. For example, suppose that an equilibrium specifies $q_{L L}^{1}=1 / 2, q_{L H}^{1}=1, q_{H L}^{1}=0$ and $q_{H H}^{1}=1 / 2$. This market-share allocation can be achieved without informative communication. The off-schedule incentive constraint might bind for a low-cost firm 1, if firm 1 were to know that firm 2 has low cost: it would then be tempting to cut price slightly and pick up the remaining $1 / 2$ of the market. By contrast, if a low-cost firm 1 were unaware of firm 2's cost type, then the off-schedule incentive constraint would be more easily met, since firm 1 then already anticipates winning the entire market with probability $\eta_{H}$. In this way, the absence of informative communication can promote cooperation, by preserving uncertainty about opponent play and thereby softening the off-schedule incentive constraint. ${ }^{40}$ Notably, uninformative communication relieves some of the pressure to give up productive efficiency that is present in an informative PPE, since it becomes easier to lower $q_{L L}^{1}$ while maintaining $q_{L H}^{1}=1$ without violating the off-schedule constraints.

We turn now to a formal representation of the incentive constraints under uninformative communication. With appropriate definitions, we can represent the

inally, let $v_{j k}^{1}$ represent the continuation value for firm 1 that is induced by the price selections $\rho^{1}\left(\theta_{j}\right)$ and $\rho^{2}\left(\theta_{k}\right)$. With these definitions in place, the on-schedule incentive constraints are again represented by IC-Oni $i_{D}$ and ICOni $i_{U}$. To define the off-schedule constraints, it is somewhat easier to refer directly to the decentralized pricing strategies. For $j \in\{L, H\}$, IC-Off $1_{j}^{U}$ is defined as:

$$
\begin{aligned}
& \sum_{k \in\{L, H\}} \eta_{k}\left[q_{j k}^{1}\left(p_{j k}-\theta_{j}\right)+\delta v_{j k}^{1}\right] \\
\geq & \max \left(\left(\rho^{2}\left(\theta_{L}\right)-\theta_{j}\right), \eta_{H}\left(\rho^{2}\left(\theta_{H}\right)-\theta_{j}\right), 0\right)+\delta \underline{v}^{1},
\end{aligned}
$$

while the corresponding constraint for firm 2 , IC-Off2 $2_{k}^{U}$, is defined analogously.

For a given continuation-value set $V$, we now define a function $C(\mathbf{p}, \mathbf{q}, \mathbf{v})$, where $C$ : $\Re_{+}^{4} \times[0,1]^{4} \times V^{4} \rightarrow\{0,1\}$. We let $C(\mathbf{p}, \mathbf{q}, \mathbf{v})=1$ if the specified prices, market shares or continuation values require informative communication, while $C(\mathbf{p}, \mathbf{q}, \mathbf{v})=0$ if there exists decentralized strategies $\left(\alpha^{i}\left(\theta^{i}\right)=N, \rho^{i}\left(\theta^{i}\right), \varphi^{i}\left(\theta^{i}\right)\right)$ that can induce the specified prices,

\footnotetext{
${ }^{40}$ In other words, when firms do not communicate, they can pool off-schedule incentive constraints across different information states. This is broadly analogous to Bernheim and Whinston's (1990) finding that multi-market contact between perfectly-informed firms serves to pool incentive constraints and facilitate cooperation.
} 
market shares and continuation values. Notice that continuation values can only be statecontingent to the extent that the state of the world is revealed by pricing and market-share divisions. When $C(\mathbf{p}, \mathbf{q}, \mathbf{v})=1$, the off-schedule constraints defined above, IC-Off $i^{I}$ and IC-Off-M $i$, are appropriate, while the IC-Off ${ }^{U}$ constraints are appropriate if $C(\mathbf{p}, \mathbf{q}, \mathbf{v})=0$.

The feasible set for when firms use uninformative communication can be written as follows:

$$
\mathcal{F}^{U}(V)=\left\{\begin{array}{c}
\mathbf{z}=(\mathbf{p}, \mathbf{q}, \mathbf{v}) \in \mathcal{Z}(V): C(\mathbf{p}, \mathbf{q}, \mathbf{v})=0 ; \text { For all } i=1,2, j \in\{L, H\}, \\
\text { IC-Oni } i_{D}, \text { IC-On } i_{U} \text { and IC-Off } i_{j}^{U} \text { hold. }
\end{array}\right\}
$$

Notice that, since the off-schedule constraints are different from the case of informative PPE, neither $\mathcal{F}^{U}(V)$ nor $\mathcal{F}^{I}(V)$ is a subset of the other. The set of uninformative PPE can then be characterized as the largest invariant set of the following operator:

$$
\tilde{T}^{U}(V)=\left\{\begin{array}{c}
\left(u^{1}, u^{2}\right): \exists \mathbf{z}=(\mathbf{p}, \mathbf{q}, \mathbf{v}) \in \mathcal{F}^{U}(V) \\
\text { such that for } i=1,2, u^{i}=\bar{U}^{i}(\mathbf{z}) .
\end{array}\right\}
$$

An initial observation is that in an uninformative PPE, even if firms collude at the reservation price and the off-schedule constraints are slack, the set of feasible policy vectors $\mathcal{F}^{U}(V)$ is not convex. Thus, the set of equilibrium values may not be convex, so that we rely more heavily on our assumption that firms can randomize among continuation equilibria. ${ }^{41}$

We now discuss several circumstances under which restricting communication might hurt firms if the off-schedule constraints do not bind. First consider the choice of $q_{L H}^{1}$. In regions where the continuation value frontier is too steep or too flat, or if the continuation value frontier is too narrow, Propositions 8 and 9 establish that the firms implement productive inefficiency. In such cases, intermediate values of $q_{L H}^{1}$ are typically optimal, so that the restriction to uninformative communication may be costly. The computational examples in Figures 3 and 4 illustrate a range of equilibrium values where, for the $(\mathbf{p}, \mathbf{q}, \mathbf{v})$ that implement these values, $C(\mathbf{p}, \mathbf{q}, \mathbf{v})=1$. In Figure 4 , the $(\mathbf{p}, \mathbf{q}, \mathbf{v})$ that implement the middle states clear the off-schedule constraints with slack, and thus restricting attention to $(\mathbf{p}, \mathbf{q}, \mathbf{v}) \in \mathcal{F}^{U}(V)$ unambiguously lowers cartel profits.

More formally, observe the tradeoff between productive efficiency and future inefficiency can be characterized in a manner analogous to Proposition 8. A scheme $(\mathbf{p}, \mathbf{q}, \mathbf{v})$ where $q_{L H}^{1}=1 / 2$ can be improved upon by a scheme where $\tilde{q}_{L H}^{1}=1$ and $\tilde{v}_{L H}^{1}$ is chosen to satisfy IC-On $1_{D}$ (holding the rest of the scheme fixed), if

$$
1+\left[f\left(v_{L H}^{1}\right)-f\left(\tilde{v}_{L H}^{1}\right)\right] /\left(v_{L H}^{1}-\tilde{v}_{L H}^{1}\right)<\left(\theta_{H}-\theta_{L}\right) /\left(p_{L H}-\theta_{L}\right) .
$$

\footnotetext{
${ }^{41}$ Of course, it could be argued that randomizing between continuation equilibria is less plausible when firms cannot communicate; however, from a formal perspective, there is no need for firms to communicate on an ongoing basis in order to randomize.
} 
However, if the frontier is too narrow, or if it eventually becomes too steep, firms sacrifice productive efficiency even if (4.2) holds so that, were it available, a small increase in $q_{L H}^{1}$ (holding the market shares in other states fixed) would increase profits. Thus, restricting communication can lead to greater productive inefficiency. Similarly, it would often be optimal to choose intermediate values of $q_{H H}^{1}$ and $q_{L L}^{1}$, typically not equal to one another, if those were available.

Thus, we have argued that there exist circumstances under which restricting communication to be uninformative lowers the profits available to the cartel. A second question concerns whether these losses accrue for every discount factor, or whether the losses disappear when firms are sufficiently patient or impatient. Clearly, restricting communication does not hurt in the static Nash equilibrium, or if the firms are too impatient to implement nonstationary market shares. Restricting communication also cannot hurt if the firms are patient enough to implement first-best in an uninformative PPE. The following proposition establishes that, even when communication is prohibited, we still have the result that there exists a critical discount factor strictly less than 1 above which first-best is attained. In particular, the linear self-generating segment constructed in Proposition 1 can be implemented without communication.

Proposition 10. Assume that $r-\theta_{H}<\theta_{H}-\theta_{L}$, and define $\delta^{F B}$ as in Proposition 1. For all $\delta \in\left[\delta^{F B}, 1\right]$, there exists an uninformative PPE that yields first-best profits to the cartel: $\left(\pi^{F B} /(1-\delta), \pi^{F B} /(1-\delta)\right) \in V^{U}$.

Thus, restricting communication hurts collusive ventures, but only when firms are moderately patient. For further characterizations of $V^{U}$, we note that most of our results about $V^{I}$ can be modified to characterize $V^{U}$, but we will not pursue that here.

Building on these results, we turn finally to a discussion of the qualitative features of the set of unrestricted PPE, where the choice of whether or not to use informative communication can depend on history. One way to understand the game is that in each period, the firms first choose whether or not to communicate. If they communicate, they reveal their types and face the IC-Off $i^{I}$ constraints; if they do not, they choose from a restricted set of market-share and price policies, but they face the relaxed IC-Off $i$ constraints. Formally, they can select from two distinct sets of feasible policy vectors:

$$
\tilde{T}(V)=\left\{\begin{array}{c}
\left(u^{1}, u^{2}\right): \exists \mathbf{z}=(\mathbf{p}, \mathbf{q}, \mathbf{v}) \in\left\{\mathcal{F}^{U}(V) \cup \mathcal{F}^{I}(V)\right\} \\
\text { such that for } i=1,2, u^{i}=\bar{U}^{i}(\mathbf{z}) .
\end{array}\right\}
$$

Consider a qualitative example where firms choose to refrain from communication. Suppose that the firms implement productive efficiency, so that firm 1 has no incentive to deviate in state $(L, H)$, and they wish to implement a scheme that is biased in favor of firm 2. If the firms attempt to reduce the market share or continuation value of firm 1 in state 
$(L, L)$ too far, IC-Off1 ${ }_{L L}^{I}$ will bind. By refraining from communication, the firms can pool the $(L, L)$ off-schedule constraint with the non-binding $(L, H)$ off-schedule constraint, thus allowing for a lower profit for firm 1 in state $(L, L)$ (e.g., market share equal to 0 ).

More generally, our analysis suggests that firms use communication in order choose a policy vector from $\mathcal{F}^{I}(V)$, so as to implement market-sharing arrangements that are not available using decentralized schemes; however, when there is significant gain from relaxing the off-schedule constraints, and when the "ideal" market shares are close enough to a scheme that can be implemented without communication, firms refrain from communicating, choosing a policy vector from $\mathcal{F}^{U}(V)$. For example, in Figure 5, the utility pair of state 1 could be approximated using a decentralized scheme, where firm 1 always prices at $r-\varepsilon$, and firm 2 prices at $r$ when its cost is high and at $r-\varepsilon$ when its cost is low. Then, firm 2 receives no market share when its cost is high, while it splits the market with firm 1 when its cost is low. Once the off-schedule constraint is relaxed by refraining from communication, it may be possible to shift $v_{L L}^{1}$ and $v_{H H}^{1}$ to the Southeast along the frontier, producing an equilibrium utility pair to the Southeast of state 1. In turn, such an equilibrium value could be used to provide future market-share favors when implementing other states, increasing productive efficiency across the frontier.

\section{Symmetric PPE}

In this section, we explore the role of our assumption that firms have access to future market-share favors. We examine the way in which collusive behavior changes when (i). firms can communicate; (ii) no bribes are permitted; and (iii) firms are restricted to use Symmetric PPE. Although there may be only limited circumstances where firms can communicate but not track behavior over time, an examination of this possibility further clarifies the consequences of the institutional environment for collusive conduct. ${ }^{42}$ In any case, we establish that even when it is available, informative communication is always weakly dominated in a Symmetric PPE, and is strictly dominated for an intermediate range of discount factors.

Working with a continuous-type model in which communication between firms is not allowed, Athey, Bagwell and Sanchirico (1998) have previously established that the optimal symmetric collusive scheme is stationary, and, under a wide range of parameter values, all cost types charge the same price and win with equal probability. In this section, we use Lemma 5 to confirm the analogous result for the discrete-type model with communication.

Consider the content of the restriction to Symmetric PPE. Formally, the set $V^{S}$ of

${ }^{42}$ To see an example where communication might be possible, consider the common practice in art auctions of using agents to represent buyers, often anonymously. A group of agents could meet before an auction without revealing their clients. If clients can use different agents in different auctions, their identities can remain concealed. 
Symmetric PPE values is the largest invariant set of the operator $T^{S}(V)$, which imposes the additional restriction $u^{1}=u^{2}$ on the set of values permitted. Applying the logic of Lemma 2, we seek to analyze the operator

$$
\tilde{T}^{S}(V)=\left\{\begin{array}{c}
\left(u^{1}, u^{2}\right): \exists \mathbf{z}=(\mathbf{p}, \mathbf{q}, \mathbf{v}) \in\left\{\mathcal{F}^{U}(V) \cup \mathcal{F}^{I}(V)\right\} \\
\text { such that for } i=1,2, u^{1}=u^{2}=\bar{U}^{i}(\mathbf{z}) .
\end{array}\right\}
$$

Consider a set $V$ that is closed and bounded. Clearly, any $V$ satisfying $u^{1}=u^{2}$ for all $\left(u^{1}, u^{2}\right) \in V$ is contained in $V^{\prime}=\left\{\left(u^{1}, u^{2}\right):\left(u^{1}, u^{2}\right) \square \sup \{u \in V\}\right\}$. Thus, we can apply Lemma 5 to characterize the best point in $\tilde{T}^{S}(V)$, since Lemma 5 implies that even with a larger set $V$ available, the firms simply use the best continuation values in the set regardless of the state. Formally, Lemma 5 indicates that the largest point in $\tilde{T}^{S}(V)$ is implemented using $\max \left\{\left(u^{1}, u^{2}\right) \in V\right\}$ as a continuation value, corresponding to a stationary equilibrium of the repeated game (i.e. $v_{j k}=\max V^{S}$ for all $j, k$ ). Further, since the schemes of Lemma 5 can be implemented without informative communication, the firms choose not to communicate.

Proposition 11. There exists a $\delta^{s}<1$ such that, for all $\delta \in\left(\delta^{s}, 1\right]:{ }^{43}$ (i) If (Pool) holds, then the optimal Symmetric PPE is stationary, and it yields value to each firm of $u^{i}=(r-$ $E[\theta]) /(1-\delta)$. This is inplemented as follows: each firm prices at $r$ in every period, communication is uninformative and the firms divide the market evenly. (ii) If (Pool) fails, then the optimal Symmetric PPE yields values $u^{i}=\left(\eta_{H}\left(r-\theta_{H}\right)+\eta_{L}\left(1+\eta_{H}\right)\left(\theta_{H}-\theta_{L}\right)\right) /(2(1-\delta))$. This can be implemented using a stationary equilibrium with uninformative communication and the pricing scheme outlined in Lemma 5.

Since informative communication is not necessary to implement the best collusive schemes, it provides no benefit; it simply tightens the off-schedule incentive constraints. Thus, there exist discount factors under which maximal collusive profits can be implemented only if firms refrain from informative communication.

The optimal equilibria for less patient firms are analyzed in greater depth in Athey, Bagwell and Sanchirico (1998), so here we simply highlight a few brief points. When (Pool) holds, the low-cost type is most tempted to deviate from the rigid-price rule. When $\delta<\delta^{s}$, it is necessary to "separate" the cost types and specify a lower price for a lowcost firm, in order to mitigate the incentive of such a firm to undercut. ${ }^{44}$ In this case, (Pool) implies that the collusive profit is lower. In the computational example of Figure 5,

${ }^{43}$ In particular, when (Pool) holds, $\delta^{s}$ satisfies $\frac{\delta^{s}}{1-\delta^{s}} \frac{r-E \theta}{2}=\frac{1}{2}\left(r-\theta_{L}\right)+\delta^{s} \underline{v}^{1}$. When (Pool) fails, the expression is more complicated (but straightforward to compute given a set of parameter values), since different off-schedule constraints might bind first for different parameter values.

44 This logic, which is developed further in Athey, Bagwell and Sanchirico (1998), is reminiscent of arguments made by Rotemberg and Saloner (1986), although they emphasize that all firms must reduce price when a high and public demand shock is encountered. 
$\delta=\delta^{s}=.71 ;$ yet, in the self-generating set of informative PPE values depicted in Figure 5 , the firms are able to achieve partial productive efficiency, and thus strictly greater profits, than in the best Symmetric PPE. Similarly, the linear self-generating set constructed in Section 4.2 exists for $\delta>.7001$, and this scheme also improves upon the rigid-price rule. From the perspective of policy, we therefore conclude that regulations that might appear to hinder collusion, such as withholding the identities of the bidders in a procurement auction, might have the perverse consequence of leaving prices unchanged but lowering productive efficiency.

\section{Bribes}

In this section, we extend the model to allow for bribes, and we refer to the extended model as the Bribes model. The following stage is added to the extensive form stage game: (v) firm $i$ sends $b^{i} \geq 0$ to firm $j$; firm $j$ receives $\gamma b^{i}$. Observe that (ex ante) communication as defined in our model is not necessary to implement bribes; the firms can condition bribes on the market shares realized ex post. To simplify the exposition, we restrict attention to informative communication; however, our main results carry over to endogenous communication. The exogenous parameter $\gamma \in[0,1]$ describes the inefficiency of the bribe; $\gamma=1$ corresponds to the use of money withou

incentive constraints, we first observe that optimal collusion never requires a state in which both firms send bribes, since, with $\gamma \square 1$, the desired net transfer can be achieved most efficiently if a single bribe is made from one firm to the other. With this observation imposed, and under informative communication, the off-schedule constraint for firm 1 can be written as:

$$
\gamma b_{j k}^{2}-b_{j k}^{1}+\delta\left(v_{j k}^{1}-\underline{v}^{1}\right) \geq \max \left(q_{j k}^{2}\left(p_{j k}-\theta_{j}\right), q_{j k}^{1}\left(\theta_{j}-p_{j k}\right)\right) .
$$

IC-Off $2_{j k}^{B}$ is defined analogously; likewise, IC-Off-M $i^{B}$ is constructed from IC-Off-M $i$ in the natural way. Notice that when IC-Off1 ${ }_{j k}^{B}$ holds, if firm 1 is assigned to send a bribe to firm 2, it never has the incentive to withhold the bribe after production takes place. After 
production takes place, firm 1 wishes to adhere to the equilibrium play if $\gamma b_{j k}^{2}-b_{j k}^{1}+\delta\left(v_{j k}^{1}-\right.$ $\left.\underline{v}^{1}\right) \geq 0$. But this inequality holds by IC-Off $1_{j k}^{B}$. In words, it is more tempting to deviate from the agreement before production takes place, as the deviating firm can capture the market and avoid paying a bribe if that is required, than after production takes place, when the firm can only avoid paying the bribe.

Let $\mathcal{F}^{I B}(V)$ be defined as $\mathcal{F}^{I}(V)$, once the utility functions and constraints from the base model are replaced with the utility functions and constraints with bribes. The policy vector is now $(\mathbf{z}, \mathbf{b})$, where $\mathbf{z}=(\mathbf{p}, \mathbf{q}, \mathbf{v})$. Finally, with ex ante utility given as $\bar{U}^{B i}(\mathbf{z}, \mathbf{b})$, let

$$
\tilde{T}^{B}(V)=\left\{\begin{array}{c}
\left(u^{1}, u^{2}\right): \exists(\mathbf{p}, \mathbf{q}, \mathbf{v}, \mathbf{b}) \in \mathcal{F}^{I B}(V) \\
\text { such that for } i=1,2, u^{i}=\bar{U}^{B i}(\mathbf{z}, \mathbf{b}) .
\end{array}\right\}
$$

We denote the set of PPE values in the Bribes model as $V^{B}$, which following our previous arguments is the largest invariant set of $\tilde{T}^{B}$. Let $v_{s}^{B}$ be the point on the Pareto frontier of $V^{B}$ that gives equal utility to both agents.

Proposition 12. (i) Suppose that $\left(r-\theta_{L}\right) /(2 \delta)<v_{s}^{1 B}-\underline{v}^{1}$. Then for all $\gamma<1$, if any productive efficiency is implemented $\left(q_{L H}^{1}>1 / 2\right)$, the equilibrium is non-stationary. If $\gamma=$ 1 , there exists a non-stationary $(\mathbf{p}, \mathbf{q}, \mathbf{v})$ that implements $v_{s}^{B}$. (ii) Assume $r-\theta_{H}<\theta_{H}-\theta_{L}$. For all $\gamma<(=) 1$, there exists $\delta^{B}<1$ such that, for all $\delta \in\left[\delta^{B}, 1\right]$, bribes are never used (resp. not necessary) along the equilibrium path in the most profitable equilibria for the firms.

Proposition 12 examines the extent to which bribes replace market-share favors in implementing Pareto efficient equilibrium values. Recall that we have already established that, so long as the off-schedule constraints do not bind at the symmetric point on the Pareto frontier of the set of informative PPE values, the frontier has an open interval of slope -1 . Thus, it is initially more efficient to use market-share favors than inefficient bribes. Further, when sufficiently patient firms can attain first-best without bribes, inefficient bribes are dominated. ${ }^{45}$

Proposition 13. Fix $\delta$ and $\gamma$, and suppose that $(\mathbf{p}, \mathbf{q}, \mathbf{v}, \mathbf{b})$ implements a Pareto efficient value $\mathbf{u} \in V^{B}$. Suppose that $p_{H L}, p_{L H}>\theta_{H}$. If $1-\gamma<\left(\theta_{H}-\theta_{L}\right) /\left(p_{L H}-\theta_{L}\right)$ and $1-\gamma<\left(\theta_{H}-\theta_{L}\right) /\left(p_{H L}-\theta_{L}\right)$, then the scheme uses productive efficiency.

Proposition 13 characterizes the use of bribes to provide incentives for productive efficiency, when firms are not patient enough to implement first-best. Bribes can expand the set of informative PPE values, whenever the equilibrium without bribes calls for the

\footnotetext{
${ }^{45}$ In a continuum-type model, the Pareto frontier has slope equal to -1 at the Pareto efficient equilibrium providing equal utility to both firms, if firms are sufficiently patient such that the off-schedule constraints permit small future market-share favors. Thus, for any $\gamma<1$, even in a continuum-type model, bribes do not fully replace future market-share favors.
} 
use of continuation values that entail greater future inefficiency than the inefficiency of bribes. Figure 6 illustrates how bribes augment the set of continuation values available to the firms. Recall that Proposition 8 establishes that firms use productive efficiency unless the continuation values are not available or require too much future inefficiency, even if the off-schedule constraints bind.

Thus, so long as bribes are suitably efficient, firms use productive efficiency. This analysis highlights an important theme: the main factor limiting productive efficiency of firms is the availability of an instrument for transferring utility, rather than off-schedule constraints. Intuitively, in the absence of bribes, if firms achieve productive efficiency today, then a market-share transfer may be required tomorrow; therefore, tomorrow's marketshare allocation is burdened with twin objectives: it must embody the required transfer for today's productive efficiency, and, ideally, it also must be productively efficient itself. As we have shown in Section 4, these objectives can sometimes clash. When bribes are available, however, they can be used to achieve the first objective, leaving tomorrow's market-share allocation free to achieve the second. Bribes can thus enable a substantial improvement in productive efficiency for firms attempting to effect transfers, provided that the associated transactions cost $(1-\gamma)$ is low enough.

As a point of contrast, now consider the use of bribes in the case of Symmetric PPE. Let $T^{B S}(V)$ be defined from $T^{B}(V)$, imposing the additional constraint that $u^{1}=u^{2}$, and let $V^{B S}$ be the largest invariant set of $T^{B S}$. From the perspective of designing collusive agreements, we can think of bribes as enlarging the set of continuation values beyond $V^{S}{ }^{46}$ Using the lemmas from Section 4.4, we have:

Proposition 14. (i) The Pareto Optimal equilibrium value in $V^{B S}$ can be implemented using a stationary collusive scheme; (ii) If $1-\gamma<\left(\theta_{H}-\theta_{L}\right) /\left(r-\theta_{L}\right)$ and firms are sufficiently patient, the optimal collusive scheme is stationary, and it entails productive efficiency, pricing efficiency and the use of bribes.

Although we do not include the computation of the critical discount factor for supporting efficiency in this model, it is important to note that this optimal collusive scheme can be implemented without informative communication. When the firms do not communicate, it can be easily shown that the critical discount factor for sustaining collusion with productive and pricing efficiency when $1-\gamma<\left(\theta_{H}-\theta_{L}\right) /\left(r-\theta_{L}\right)$ is strictly lower than the critical discount factor for sustaining rigid prices and productive inefficiency when $\gamma=0$.

These results have two main implications for applied analysis of collusion. First, we observe that firm-specific reputations and non-stationarity (i.e., market-share favors) are

\footnotetext{
${ }^{46}$ In terms of applications, there may be only limited circumstances where firms cannot track individual behavior over time, but they can use individual-specific bribes as conditioned on current-period announcements; however, an cxample is an auction market where the bidders use agents (as is common in art auctions), agents represent multiple bidders and bidders can switch agents without observation.
} 
robust features of collusive ventures, so long as bribes are inefficient and individual firm behavior can be tracked over time. Non-stationary equilibria (including on-equilibriumpath price wars) are not useful in two extreme cases: legalized cartels with side-payments and cartels facing informational or other (unmodelled) constraints that force them into Symmetric PPE. ${ }^{47}$

Second, our results have a somewhat perverse policy implication. To the extent that bribes are used, in either symmetric or asymmetric PPE, they increase the productive efficiency of the cartel. For many discount factors and parameter values, firms can sustain collusion at high prices, and the only issue for the cartel is the extent to which they can implement productive efficiency. Thus, for many discount factors, prohibiting bribes reduces welfare. On the other hand, there do exist moderate discount factors such that prohibiting bribes lowers the collusive profits enough that collusion takes place only at substantially lower prices. For moderately patient firms, therefore, a prohibition on bribes may raise consumer welfare.

\section{Policy and Empirical Implications}

Our analysis has a variety of implications for policy. One theme is that successfully colluding firms tolerate productive inefficiency before lowering prices. ${ }^{48}$ From the perspective of welfare, that is bad news: policies that limit firms' ability to communicate and use bribes may serve mainly to limit productive efficiency, without having a large effect on prices. These policies increase consumer welfare only if firms are sufficiently impatient that removing these instruments destroys their ability to collude at high prices. ${ }^{49}$ Further, prohibiting bribes may not have much effect at all, unless the firms have moderate patience. Overall, our findings therefore provide some formal support for those (e.g., Bork $(1965,1966)$, Sproul (1993)) who are attentive to the possible efficiency gains that restraints of trade may afford, and who therefore question the unqualified application of the per se rule against price fixing. ${ }^{50}$

Similar results hold when we consider designing markets (such as auction markets for

${ }^{47}$ Of course, in reality, tracking a complicated, non-stationary agreement, and potentially communicating about cost information, also has costs, so our comparison between inefficient bribes and future marketshare favors may be biased against bribes; nonetheless, our results indicate that market-share favors can be quitc effective as a collusive mechanism for sufficiently patient firms.

${ }^{48}$ This theme arises in the base model, as a comparison of Propositions 6 and 9 confirm. It also appears when we consider policies that prohibit communication (see the discussion preceding Proposition 10), impose anonymity (see Propositions 11 and 14) or prohibit bribes (see Propositions 13 and 14).

${ }^{49}$ Of course, in our inelastic-demand model, social welfare is independent of price. Qualitatively similar findings would also arise in a model with downward-sloping demand, however.

50 At the same time, we notc that our findings depend somewhat on the ability of firms to use small differences in prices to allocatc market share. As discussed in footnote 38, in a Cournot model, restricting communication might improve consumer welfare. 
government contracts or internet auction markets) to withhold firm identities. ${ }^{51}$ If firms are patient enough to support the fixed-price equilibrium, policies that impose anonymity only reduce productive efficiency. On the other hand, there are discount factors under which the best Symmetric PPE entails productive efficiency and lower prices when firms have low cost, while the best asymmetric PPE entails some productive inefficiency but monopoly prices.

Another implication of our model concerns merger policy. Although our formal model considers only two firms that draw from identical cost distributions, the modeling framework can be easily extended to allow for multiple firms with asymmetric cost distributions. As is customary, with more firms, the critical discount factor for supporting cooperation goes up. But asymmetries also frustrate collusion among impatient firms: a firm who expects higher cost and/or lower market share in the future is more tempted to deviate from a collusive agreement. It thus may be more difficult for asymmetric firms to implement future market-share favors. Accordingly, a merger that creates asymmetries across firms may impede the provision of market-share favors and thereby the attainment of productive efficiency.

In terms of empirical implications, our results suggest the following taxonomy: very impatient firms use low prices that vary significantly with costs, and implement productive efficiency; slightly more patience leads to higher prices, but still prices that vary with cost; firms who are more patient still, but who face informational or coordination constraints that prevent tracking individuals over time, use high prices that do not vary with cost, and each firm's market share is independent over time; and finally, firms who are patient and sophisticated use high prices that do not vary with cost, but they implement productive efficiency and a firm's market share is negatively correlated over time. An implication of our analysis is thus that market-share volatility and productive efficiency are non-monotonic in the "extent" of collusion, being greatest when firms interact noncooperatively or employ sophisticated collusive schemes.

Our results also provide some insight into the role of institutions, such as industry associations, industry trade publications and the celebrated "smoke-filled room." We identify a potential benefit to communication: it allows firms to allocate market share in a statecontingent fashion. Clearly, this only has value in collusive schemes that implement some productive efficiency. ${ }^{52}$ On the other hand, we have seen that firms may prefer to avoid

\footnotetext{
${ }^{51}$ Indeed, an interesting question for policy concerns the design of formal auction markets: should the auctioneer reveal the identities of the bidders? For example, an internet market can be designed using aliases, or it can be designed so that participants are required to publicly reveal their identities. In the former case, the market-maker provides the service of allowing participants to meet anonymously and still conduct transactions; in the latter case, the market-maker provides the scrvice of verifying identities (for example, using credit cards).

52 In alternative models, communication might offer other benefits; for example, firms might share information to better forecast future demand.
} 
communication following some histories, in order to keep impatient firms from deviating after they learn that they will not produce. We have also provided some negative results about the use of bribes; they are mainly useful for firms of moderate patience, or firms who expect difficulty in tracking identities over time. They typically do not fully replace marketshare favors as a mechanism for providing incentives, and thus an optimally-designed cartel allocates current production in a manner that is sensitive to firm-specific histories.

Our results provide a lens to guide future empirical and case-based research about collusive behavior. However, market-share favors may be difficult to document, since they do not necessarily generate the same kind of "paper trail" as might a series of side-payments. Indeed, most of the detailed case studies of cartels come from historical examples of legalized cartels where side-payments were permitted (see, e.g., Stocking and Watkins (1946)). But there are more recent examples as well. For example, McMillan (1985) describes collusion among firms in the $\mathrm{J}$

ith our formal analysis, McMillan reports that firms use future market-share favors, and sometimes even bribes, as a means of providing incentive for honest communication so that greater productive efficiency can be achieved.

With the recent increase in anti-trust enforcement of price-fixing cases in the U.S. (and the incentives it provides to conspirators to divulge details of the workings of the cartel),$^{53}$ it may be possible to gain further insight into the institutional design of individual contemporary cartels and to document the kinds of behavior studied in this paper. In many of the recent cases, market-share allocation was a primary concern of the cartel; examples include citric acid, feed additives, marine services, graphite electrodes, and vitamins. ${ }^{54}$ These cartels differed in their sophistication and design. According to prosecutors, the main topic of explicit communication for these cartels, particularly among high-level executives, appeared to be market-share allocation. Some of the cartels, such as marine services, allocated individual customers and used side-payments and profit-sharing, following a written set of rules. Interestingly, other cartels used a mechanism closer in spirit to our market-share favors: in the case of citric acid, cartel members examined market shares at the end of each year, and any company who sold more than a pre-specified amount had to make it up in the following year by purchasing from other cartel members whose market shares fell below the target.

More generally, the existing literature on collusion typically does not distinguish between market-share allocation schemes that implement productive efficiency and those that use pre-determined (and thus inefficient) allocation rules. For example, in the case of bid-

${ }^{53}$ The U.S. Justice Department began a "leniency" program in 1993, whereby the first conspirator in a price-fixing case to confess is immune from criminal charges.

${ }^{54}$ Sce Business Week (July 27, 1998), "Justicc's Cartel Crackdown," pp. 50-51 for more discussion. 
rigging at auctions, bid rotation schemes are common, but many of the famous examples involve randomized market-share allocation (i.e., "Phases of the Moon"). Comanor and Schankerman (1975) analyzed all prosecuted cases of bid rigging over a 50-year period, and they showed that cartels with a smaller number of bidders were more likely to use bid rotation schemes than "identical bidding." Although they did not provide much detail about the nature of the bid rotation, further study of the legal testimony may allow us discern those rotation schemes that made use of market-share favors or bribes to implement productive efficiency. In other applications, it may be possible to verify the prediction of negative correlation in the market shares of firms using sophisticated rotation schemes as opposed to randomized rotation schemes.

\section{Conclusions}

While the results in this paper are motivated by the problem of collusion, they can be applied to a number of other contexts. At a general level, our model considers interactions between agents - such as family members, workers in a firm or politicians - who must repeatedly take actions in an environment with two main characteristics: first, each firm's cost or benefit of taking the action changes from period to period, and the actual change is private information; and second, there are limits on the firms' ability to use explicit sidepayments. Essentially, the repeated play of any of the standard multi-agent mechanism design problems (public goods, auctions, bargaining) fits into the framework, with the additional assumption of restricted transfers.

Private information is easy to motivate in many contexts. For example, family members may be privately informed about how tired they are on a particular day, and thus how costly it is to perform household work. Likewise, division heads within a firm may have private information about the efficiency of access to a resource, and politicians may have private information about the costs of legislation. Limits or transaction costs associated with transfers also arise frequently: families may share a common budget, division heads may share a common resource and payments for votes may be illegal. Social norms may also prohibit monetary transfers in certain circumstances. In this broader context, our paper can be interpreted as providing a formal analysis of the hierarchy of relationships available, as well as the extent of efficiency achieved by each type of relationship. For example, we establish that the promise of future favors may eliminate the need and desire of agents to use inefficient bribes in the present. ${ }^{55}$

\footnotetext{
${ }^{55}$ In this regard, our results are similar to those of Holmstrom and Kreps (1996), who study the use of "promises" as opposed to monetary payments for favors. Our analysis differs from theirs in that we bring together the tools of dynamic programming and mechanism design to characterize optimal equilibria for firms for a given discount factor, and we explicitly model the tradeoff between different kinds of sidepayments.
} 
¿From a methodological perspective, our analysis highlights several points. First, our paper is the first of which we are aware to provide tools for characterizing the optimal use of market-share favors by impatient firms. The problem of cartel design motivates our comparison between a variety of instruments available to the colluding firms, and impatient firms face real tradeoffs among those instruments. We identify these tradeoffs and explore them both theoretically as well as using computational examples, showing that it is possible to provide rich characterizations of optimal collusive schemes. Second, we develop the precise connections between static and dynamic analyses of collusion, making clear the similarities and differences, and laying the groundwork for treating other repeated-game problems within the mechanism design framework. Third, our work motivates some new questions for static mechanism design, and takes some initial steps towards addressing them. ${ }^{56}$ Finally, our comparison of symmetric and asymmetric equilibria indicates that symmetry can be a significant restriction on the set of equilibria, much as restricting or eliminating side-payments in static mechanism design problems yields drastically different answers than allowing for budget-balanced side payments. This observation motivates more careful exploration of the classes of environments suited to the two kinds of equilibria.

\section{Appendix}

Proof of Lemma 2: $\quad$ For any $\left(u^{1}, u^{2}\right) \in T^{I}(V)$, there exist $\mathrm{s} \in S$ and $v: A^{2} \times \Re^{4} \rightarrow \operatorname{co}(V)$ that satisfy incentive compatibility conditions and yield $u^{i}=\bar{\pi}^{i}(\mathbf{s})+\delta v^{i}(\mathbf{s}(\theta))$. As these functions uniquely define prices, market shares and continuation values in each state of the world, we can define the associated $(\mathbf{p}, \mathbf{q}, \mathbf{v})$. It is straightforward to verify that any $(\mathbf{p}, \mathbf{q}, \mathbf{v})$ that can be attained in the extensive-form game is in the feasible set $\mathcal{Z}(V)$, up to payoff-irrelevant choices (for example, prices greater than $r$ that receive no market share). It then remains to verify that the incentive constraint in $T^{I}(V)$ implies that the on- and off-schedule incentive compatibility constraints of $\mathcal{F}^{I}(V)$ are satisfied. Any desired on-schedule deviation can be achieved by using $\tilde{\alpha}^{i}\left(\theta^{i}\right) \neq \theta^{i}$, while any off-schedule deviation can be achieved by choosing the appropriate $\tilde{\alpha}^{i}$ and $\tilde{\rho}^{i}$. Thus, $(\mathbf{p}, \mathbf{q}, \mathbf{v}) \in \mathcal{F}^{I}(V)$, and thus $\left(u^{1}, u^{2}\right) \in \tilde{T}^{I}(V)$. Now consider a $\left(u^{1}, u^{2}\right) \in \tilde{T}^{I}(V)$. Again, any $(\mathbf{p}, \mathbf{q}, \mathbf{v}) \in \mathcal{Z}(V)$ can be achieved with the appropriate $\mathbf{s} \in S$ and $\boldsymbol{v}: A^{2} \times \Re^{4} \rightarrow \operatorname{co}(V)$. The on-schedule incentive constraints imply that $\alpha^{i}\left(\theta^{i}\right)=\theta^{i}$, and the off-schedule incentive constraints imply that the most profitable deviations, $\tilde{\alpha}^{i}, \tilde{\rho}^{i}, \tilde{\varphi}^{i}$, give lower profits than the proposed equilibrium.

Proof of Lemma 4: Imposing pricing efficiency, productive efficiency and Pareto efficient

${ }^{56}$ For example, we examine how restrictions on transfers (for instance, to a convex set) affect optimal mechanisms. In the litcrature on collusion, only a limited class of restrictions on transfers have received attention. Cramton and Palfrey (1990) and Kihlstrom and Vives (1992) study the role of participation constraints; whilc McAfee and McMillan (1992) distinguish between "weak" and "strong" cartels, where weak cartels cannot implement any sidc-payments and strong cartels can write cnforceable contracts and makc side-payments. The model of Symmetric PPE can be interpreted as a generalized model of wcak cartels, in which firms can impose symmetric "pcnalties" in response to the placement of a low bid in a procurement auction. The strong-cartel model is closer in spirit to the model of Asymmetric PPE: transfers can be made from one firm to another, but only imperfectly, using asymmetric continuation equilibria. 
continuation values (i.e., $v_{j k}^{1}+v_{j k}^{2}=2 K$ for all $(j, k)$ ), it is straightforward to show that IC-On $1_{D}$ and $\mathrm{IC}-\mathrm{On} 2{ }_{D}$ respectively bind if and only if

$$
\begin{aligned}
& 0=\left[r-\theta_{H}\right]\left\{\eta_{H}\left(q_{H H}^{1}-1\right)-\eta_{L} q_{L L}^{1}\right\}+\delta\left\{\eta_{H}\left(v_{H H}^{1}-v_{L H}^{1}\right)+\eta_{L}\left(v_{H L}^{1}-v_{L L}^{1}\right)\right\}, \text { and } \\
& 0=-\left[r-\theta_{H}\right]\left\{\eta_{H} q_{H H}^{1}+\eta_{L}\left(1-q_{L L}^{1}\right)\right\}-\delta\left\{\eta_{H}\left(v_{H H}^{1}-v_{H L}^{1}\right)+\eta_{L}\left(v_{L H}^{1}-v_{L L}^{1}\right)\right\}
\end{aligned}
$$

Adding the constraints yields the necessary condition $v_{H L}^{1}-v_{L H}^{1}=\left(r-\theta^{H}\right) / \delta$, and we may choose the remaining market shares and continuation values to respect the additional conditions in the lemma while satisfying each of the above constraints.

Proof of Lemma 5: We posit that the downward IC-On constraints bind, and substitute in from (3.1) for $U^{i}(L, L ; \mathbf{z})$. We solve a relaxed program:

$$
\begin{gathered}
\max _{\substack{q_{L L}^{2}, q_{L H}^{2}, q_{H H}^{2}, q_{H L}^{2} \in[0,1] \\
p_{L H}, p_{H H}, p_{H L} \square r ; \bar{v}_{H}^{1}, \bar{v}_{H}^{2} \square K}} \sum_{j \in\{L, H\}} \eta_{j} \cdot q_{j H}^{2} \cdot\left(p_{j H}-\theta_{H}\right)+\delta \bar{v}_{H}^{2}+\eta_{L} \cdot \bar{q}_{L}^{2}\left(\theta_{H}-\theta_{L}\right) \\
+\lambda\left[\sum_{k \in\{L, H\}} \eta_{k} \cdot\left(1-q_{H k}^{2}\right) \cdot\left(p_{H k}-\theta_{H}\right)+\delta \bar{v}_{H}^{1}+\eta_{L} \cdot\left(1-\sum_{k \in\{L, H\}} \eta_{k} q_{L k}^{2}\right)\left(\theta_{H}-\theta_{L}\right)-u^{1}\right] \\
+\psi^{1} \cdot\left[\sum_{k \in\{L, H\}} \eta_{k} q_{H k}^{2}-\sum_{k \in\{L, H\}} \eta_{k} q_{L k}^{2}\right]+\psi^{2} \cdot\left[\bar{q}_{L}^{2}-\bar{q}_{H}^{2}\right]
\end{gathered}
$$

Let $\lambda$ be the multiplier on firm 1's utility constraint, which is non-negative on the Pareto frontier. The multipliers on the monotonicity constraints are denoted $\psi^{i}$, and these are also non-negative. By inspection, it is clearly optimal to set $p_{H k}=r, p_{j H}=r$ and $\bar{v}_{H}^{i}=K$; then, differentiating with respect to the market-share variables, we get:

$$
\begin{gathered}
\frac{\partial}{\partial q_{L L}^{2}}:(1-\lambda) \cdot \eta_{L}^{2}\left(\theta_{H}-\theta_{L}\right)-\psi^{1} \eta_{L}+\psi^{2} \eta_{L} ; \frac{\partial}{\partial q_{H H}^{2}}:(1-\lambda)\left[\eta_{H}\left(r-\theta_{H}\right)\right]+\psi^{1} \eta_{H}-\psi^{2} \eta_{H} \\
\frac{\partial}{\partial q_{L H}^{2}}: \eta_{L}\left(r-\theta_{H}\right)-\lambda \eta_{L} \eta_{H}\left(\theta_{H}-\theta_{L}\right)-\psi^{1} \eta_{H}-\psi^{2} \eta_{L} \\
\frac{\partial}{\partial q_{H L}^{2}}: \eta_{L} \eta_{H}\left(\theta_{H}-\theta_{L}\right)-\lambda \eta_{L}\left(r-\theta_{H}\right)+\psi^{1} \eta_{L}+\psi^{2} \eta_{H}
\end{gathered}
$$

(i) Notice first that if we maximize the sum of the firms' utilities $(\lambda=1)$, (Pool) implies that unless $\psi^{1}>0$ or $\psi^{2}>0$, we increase $q_{L H}^{2}$ and decrease $q_{H L}^{2}$ until the boundary constraints bind. Notice that these variables move in the direction of inefficiency. At a symmetric solution, both monotonicity constraints are binding, and there is productive inefficiency $\left(\vec{q}_{L}^{i}=\vec{q}_{H}^{i}\right)$. Now suppose that we weight the firms evenly $(\lambda=1)$ and consider asymmetric solutions. Still, there will be no productive efficiency unless $\psi^{1}+\psi^{2}>0$. Suppose that $\psi^{1}>0$ and $\psi^{2}=0$. Then, the objective is increasing in $q_{H H}^{2}$ and decreasing in $q_{L L}^{2}$, so we take $q_{H H}^{2}=1$ and $q_{L L}^{2}=0$. But then, firm 2's monotonicity constraint implies $\eta_{H}+\eta_{L} q_{L H}^{2}<\eta_{H} q_{H L}^{2}$, a contradiction.

Thus, we have established that the largest joint utility available to the firms is achieved by "pooling," where $\bar{q}_{L}^{i}=\bar{q}_{H}^{i}$, and that allowing for asymmetric allocations of utility will not improve the sum of utilities. This scheme satisfies all of the constraints in $\mathcal{F}_{O n}^{I}(V)$. So, an upper bound for the sum of utilities is given by $r-E[\theta]$. Now observe that for any $\alpha \in[0,1]$, we can allocate $\alpha(r-E[\theta])$ to firm 1 and $(1-\alpha)(r-E[\theta])$ to firm 2 by simply changing the market shares of the 
firms while maintaining $\bar{q}_{L}^{i}=\bar{q}_{H}^{i}$. Since this satisfies the IC-On constraints, the Pareto frontier is given as in the statement of the lemma.

(ii) Under the alternative assumption that (Pool) fails, inspection of the program shows that profits are highest when $q_{L H}^{2}=0$ and $q_{H L}^{2}=1$. The monotonicity constraints do not bind. At these values, the relaxed program is independent of $p_{L H}$ and $p_{H L}$. This scheme can be implemented by using pricing efficiency in state $(H, H)\left(p_{H H}=r\right), v_{j k}^{i}=K$ for all $i, j, k$, and productive efficiency. The truth-telling constraints can be satisfied as follows: find $\hat{p}<r$ to be used by all low-cost types. With $q_{L L}^{i}=q_{H H}^{i}=1 / 2$, truth-telling by a high-cost firm requires:

$$
\frac{1}{2} \eta_{H}\left(r-\theta_{H}\right)=\left(\eta_{H}+\frac{1}{2} \eta_{L}\right)\left(\hat{p}-\theta_{H}\right)
$$

yielding the price stated in the lemma. It is now direct to derive the utility frontier.

Proof of Proposition 1: We solve a set of equations for the critical discount factor. To compute $\delta^{F o n}$, consider the following system (imposing pricing efficiency, productive efficiency, and $v_{j k}^{2}=2 \pi^{F B} /(1-\delta)-v_{j k}^{1}$ for all $\left.(j, k)\right)$ : IC-On1 $1_{D}$, IC-On2 ${ }_{D}, \bar{U}^{1}=x, \bar{U}^{2}=y, v_{L H}^{1}=x$, $v_{H H}^{1}=x, q_{H H}^{1}=0$, and $q_{L L}^{1}=0$. It can be verified that under our assumption that $\eta_{L}>$ $1 / 2, v_{L H}^{1}<v_{L L}^{1}$. In particular, $v_{L L}^{1}-v_{L H}^{1}=\frac{2 \eta_{L}-1}{\eta_{L}} \cdot \frac{r-\theta_{H}}{\delta}$. Since $\frac{2 \eta_{L}-1}{\eta_{L}} \in(0,1)$, this implies $v_{L H}^{1}<v_{L L}^{1}<v_{H L}^{1}$, where the latter inequality follows since the downward on-schedule constraints imply that $v_{H L}^{1}=v_{L H}^{1}+\frac{r-\theta_{H}}{\delta}$. It remains to verify that given our solutions to these equations, $v_{H L}^{1}=x+\frac{r-\theta_{H}}{\delta}<y$. We can compute:

$$
y-x=\frac{\eta_{L}^{2}\left(\theta_{H}-\theta_{L}\right)-\left(r-\theta_{H}\right)\left(2 \eta_{L}-1\right)}{1-\delta} .
$$

This expression is always positive under our restrictions $\eta_{L}>1 / 2$ and $r-\theta_{H}<\theta_{H}-\theta_{L}$. $\delta^{\text {Fon }}$, as stated in the text, solves the equation $y-x=\frac{r-\theta_{H}}{\delta}$. It can be verified that $y-x>\frac{r-\theta_{H}}{\delta}$ for all $\delta>\delta^{F o n}$.

To compute $\delta^{\text {Foff }}$, we observe first that IC-Off $1_{L H}^{I}$ is slack, as is IC-Off-M1. Further, using the implementation described above, IC-Off $1_{H H}^{I}$ implies IC-Off $1_{H L}^{I}$. We then substitute in the values for $q_{j j}^{1}$ and $v_{j j}^{1}$ computed above, and verify (using tedious algebraic manipulation) that IC-Off1 ${ }_{L L}^{I}$ and IC-Off $1_{H H}^{I}$ are satisfied when $\delta \geq \delta^{F o f f}$, where $\delta^{F o f f}$ is given in the text. Finally, since for the endpoints of the interval, we have described a policy vector that meets all of our constraints and uses as continuation values other values on the same interval, we can then construct the remainder of the line segment using convex combinations of policy vectors to implement convex combinations of equilibrium values. This is possible because, when pricing efficiency is imposed, the constraints and utilities are linear in market shares and continuation values. Thus, we have constructed a self-generating set of equilibrium values with first-best profits to the cartel.

Proof of Proposition 2: To implement $(x, y)$, let $p_{j k}=r, v_{L H}=v_{L L}=(x, y), v_{H H}=v_{H L}$, and $q_{H L}^{1}=q_{H H}^{1}=0$. Further, set $q_{L H}^{1}=q_{L L}^{1}=\frac{\delta\left(\square+\eta_{L}^{2}\right)}{1+\square-\delta \eta_{H}}$. Finally, use the static Nash equilibrium as the off-schedule threat point. Notice first that IC-On2 $2_{D}$ and IC-On $2_{U}$ both hold with equality given these values. Given the specified market shares and off-equilibrium-path threat points, the $v_{L H}^{1}$ that satisfies IC-Off $1_{L L}^{I}$ and IC-Off $1_{L H}^{I}$ is uniquely determined. Next, it can be shown that IC-On $1_{D}$ holds with these parameter values if and only if $v_{H L}^{1}-v_{L H}^{1}=q_{L H}^{1}\left(r-\theta_{H}\right) / \delta$. Thus, it remains to verify that this distance is feasible using $v_{H L}^{1} \square y$, where $y$ is determined as firm 2's profit in this scheme. Given pricing efficiency and the specified level of productive efficiency for the cartel, and since all continuation values lie on a line with slope -1 through $(x, y)$, it is possible to compute the sum of firm profits at $(x, y), K=x+y$, as a function of the exogenous 
parameters. Then, since $v_{L H}^{1}=x, v_{H L}^{1} \square y$ holds if and only if $v_{L H}^{1}+\left(q_{L H}^{1}+q_{L L}^{1}\right)\left(r-\theta_{H}\right) /(2 \delta)$ $\square K-v_{L H}^{1}$. It can be verified that this constraint becomes relaxed as $\delta$ increases. Substituting and solving, the constraint binds for $\delta=\delta^{l i n} \equiv$

$$
\frac{\eta_{L}^{2}-\square\left(\square+\eta_{H}\right)+\sqrt{\left(\square(1+\square)-\eta_{L}\left(\eta_{L}+\square\right)\right)^{2}+4 \square(1+\square)\left(\square^{2}+\eta_{L}^{2}\left(1+\eta_{L}^{2}\right)+\square \eta_{L}\left(1+2 \eta_{L}\right)\right)}}{2\left(\square^{2}+\eta_{L}^{2}+\eta_{L}^{4}+\left[\eta_{L}\left(1+2 \eta_{L}\right)\right)\right.}
$$

Proof of Proposition 3: (i) The symmetric point of the Pareto frontier of $\tilde{T}(V)$ can be implemented with $q_{j j}^{i}=1 / 2$ and $v_{j j}^{1}=v_{j j}^{2}$. Before beginning, we observe that we can take $p_{j j}>\theta_{j}$ without loss of generality. To see why, observe that if $\theta_{j}-p_{j j}>0$, we can raise $p_{j j}$ by $\varepsilon$ and lower $v_{j j}^{i}$ by $\varepsilon /(2 \delta)$ until we arrive at $\hat{p}_{j j}$ and $\hat{v}_{j j}$, where $\theta_{j}=\hat{p}_{j j}$, without affecting any utilities or incentive constraints (since an optimal off-schedule deviation would ensure zero market-share in state $j j$ ). To see that the resulting $\hat{v}_{j j}^{i}$ is feasible, observe that given market share of $1 / 2$, our assumption that IC-Off $i_{j j}^{I}$ is slack implies that $\frac{1}{2}\left(\theta_{j}-p_{j j}\right)<\delta\left(v_{j j}^{i}-\underline{v}^{i}\right)$; since the adjustments preserve this inequality, the new continuation value $\hat{v}_{j j}^{i}$ must satisfy $\hat{v}_{j j}^{i} \geq \underline{v}^{i}$. Since the set of feasible continuation values is convex and symmetric, it is feasible. Finally, if $\theta_{j}-p_{j j}=0$, we may employ a similar adjustment, unless $v_{j j}^{i}=\underline{v}^{i}$. But in this case (OffSS) is violated.

Starting from this point, our approach is to implement an alternative utility pair, with no efficiency loss, in which $\bar{U}^{1}$ is decreased and $\bar{U}^{2}$ is increased. We define two perturbations. In Perturbation 1, we lower $q_{H H}^{1}$ by $\varepsilon /\left(\left(p_{H H}-\theta_{L}\right) \eta_{H}\right)$ and lower $q_{L L}^{1}$ by $\varepsilon /\left(\left(p_{L L}-\theta_{L}\right) \eta_{L}\right)$. For each firm $i, \mathrm{IC}-\mathrm{On} i_{U}$ is unaltered by this perturbation. In Perturbation 2, we lower $q_{H H}^{1}$ by $\varepsilon /\left(\left(p_{H H}-\theta_{H}\right) \eta_{H}\right)$ and lower $q_{L L}^{1}$ by $\varepsilon /\left(\left(p_{L L}-\theta_{H}\right) \eta_{L}\right)$. For each firm $i$, this perturbation leaves unaltered IC-Oni ${ }_{D}$. Both perturbations lower $\bar{U}^{1}$ and increase $\bar{U}^{2}$.

There are several cases to consider. Suppose first that, for a given $\lambda \in\{U, D\}$, IC-On $i_{\lambda}$ is slack for each $i$. If $\lambda=U$, we use Perturbation 2 to engineer the desired utility transfer without violating on-schedule incentive constraints. Likewise, if $\lambda=D$, we use Perturbation 1 . Next, we modify the argument for the case where the on-schedule constraints are slack in different directions. First, take the case where IC-On1 $D$ and IC-On2 $U$ are slack. If $p_{L L} \square p_{H H}$, we use Perturbation 1, which relaxes IC-On $2_{D}$ by $\left(p_{H H}-\theta_{H}\right) /\left(p_{H H}-\theta_{L}\right)-\left(p_{L L}-\theta_{H}\right) /\left(p_{L L}-\theta_{L}\right)$, which is positive by our assumption that $p_{L L} \square p_{H H}$. If $p_{L L} \geq p_{H H}$, we use Perturbation 2. This relaxes IC-On1 $1_{U}$ by $\left(p_{H H}-\theta_{L}\right) /\left(p_{H H}-\theta_{H}\right)-\left(p_{L L}-\theta_{L}\right) /\left(p_{L L}-\theta_{H}\right)$, which is positive. Similarly, in the second case, where IC-On1 $1_{U}$ and IC-On2 $2_{D}$ are slack, we proceed as follows: if $p_{L L} \square p_{H H}$, we use Perturbation 2, which relaxes IC-On2 $U$ by $\left(p_{L L}-\theta_{L}\right) /\left(p_{L L}-\theta_{H}\right)-\left(p_{H H}-\theta_{L}\right) /\left(p_{H H}-\theta_{H}\right)$, which is positive; and if $p_{L L} \geq p_{H H}$, we use Perturbation 1 , which relaxes IC-On1 $1_{D}$ by $\left(p_{L L}-\right.$ $\left.\theta_{H}\right) /\left(p_{L L}-\theta_{L}\right)-\left(p_{H H}-\theta_{H}\right) /\left(p_{H H}-\theta_{L}\right)$, which is positive.

(ii) Suppose that (a) and the first part of (b) fail: IC-Off $I_{L L}^{I}$ and IC-Off1 ${ }_{H H}^{I}$ are slack, and $v_{L L}^{1}>x$ and $v_{H H}^{1}>x$. Then, lower $v_{H H}^{1}$ by $d \varepsilon / \eta_{H}$ and lower $v_{L L}^{1}$ by $d \varepsilon / \eta_{L}$, and raise the corresponding values for firm 2 by the same amount (this is possible because $v_{j j}^{1}>x$ and the set of available continuation values is convex). This does not affect any on-schedule incentive constraints, relaxes firm 2's off-schedule constraints, and decreases $\bar{U}^{1}$ and increases $\bar{U}^{2}$ with no efficiency loss, contradicting the hypothesis that $(x, y)$ is the end point of the region with slope equal to -1 . Now consider the case where (a) and the second part of (b) fail: IC-Off $1_{L L}^{I}$ and IC-Off $1_{H H}^{I}$ are slack, $q_{L L}^{1}>0$ and $q_{H H}^{1}>0$, and $p_{j j}>\theta_{j}$ for each $j$. We will show below in Proposition 8 that under the assumptions of this proposition, $\bar{q}_{L}^{i}>\bar{q}_{H}^{i}$, which implies that for each $i$, one of IC-Oni $i_{D}$ and IC-On $i_{U}$ is slack. We may now apply the algorithm used in the proof 
of Part (i) to implement a utility pair that yields lower (higher) utility for firm 1 (2), without efficiency loss, contradicting the hypothesis that $(x, y)$ is the endpoint of a region with slope equal to -1 .

Proof of Proposition 4: (i) If each IC-Oni $i_{U}$ is slack, prices are efficient, and $q_{L H}^{1}=1$, then IC-Off1 ${ }_{L H}^{I}$ and IC-Off-M1 are slack, and we can decrease $q_{L H}^{1}$ and give the market share to firm 2 without violating any on-schedule constraints. But this makes firm 1 worse off and firm 2 better off, violating the hypothesis that the scheme implements a corner of $v_{N}^{I}$.

(ii) Suppose that (a) and the first part of (b) fail: IC-Off $1_{L L}^{I}$ and IC-Off1 $1_{H H}^{I}$ are slack, and $v_{L L}^{1}>v_{N}^{1 I}$ and $v_{H H}^{1}>v_{N}^{1 I}$. Then, for $\varepsilon_{1}$ small enough, there exists an $\varepsilon_{2}>0$ such that it is possible to lower $v_{H H}^{1}$ by $\varepsilon_{1} / \eta_{H}$ and lower $v_{L L}^{1}$ by $\varepsilon_{1} / \eta_{L}$, and raise $v_{H H}^{2}$ by $\varepsilon_{2} / \eta_{H}$ and raise $v_{L L}^{2}$ by $\varepsilon_{2} / \eta_{L}$ (this is possible because the set of available continuation values is convex and by the definition of $v_{N}^{I}$ as the north corner of the Pareto frontier). This does not upset any on-schedule constraints, and makes firm 1 worse off and firm 2 better off. There is potentially an efficiency loss, however. Next, we consider the case where (a) and the second part of (b) fail. We may then argue as in the proof of Proposition 3 and arrive at a contradiction.

Proof of Proposition 5: The proof proceeds in a series of lemmas. Part (i) follows by Lemma 7 , and part (ii) follows by Lemma 8

Lemma 6. Consider a scheme $(\mathbf{p}, \mathbf{q}, \mathbf{v})$. (T1j) If we subtract $\eta_{L} d v$ from $v_{j H}^{1}$ and add $\eta_{H} d v$ to $v_{j L}^{1}, \bar{U}^{1}, I C-O n 1_{D}$ and $I C-O n 1_{U}$ are unaffected. (T2j) If we add $\eta_{H} d v$ to $v_{L j}^{2}$ and subtract $\eta_{L} d v$ from $v_{H j}^{2}, \bar{U}^{2}, I C-O n 2_{D}$ and IC-On2 $2_{U}$ are unaffected.

Lemma 7. If $(\mathbf{p}, \mathbf{q}, \mathbf{v})$ satisfies $\mathcal{F}_{O n}^{I}(V)$ and $v_{N}^{1}>v_{j k}^{1}>v_{E}^{1}$ for all $(j, k)$, then if $v_{j k}^{2}<f\left(v_{j k}^{1}\right)$ for any $(j, k)$, this scheme is Pareto dominated by another scheme that satisfies $\mathcal{F}_{O n}^{I}(V)$, has all continuation values on the Pareto frontier of $V$ and uses the same prices.

Proof. Suppose that for some $j, v_{j H}^{2}<f\left(v_{j H}^{1}\right)$ and $v_{j L}^{2}<f\left(v_{j L}^{1}\right)$. Then, we can hold fixed firm 1's continuation values and increase $v_{j H}^{2}$ and $v_{j L}^{2}$ by the same amount without affecting IC-On2, thus increasing $\bar{U}^{2}$. Then, suppose that $v_{j H}^{2}=f\left(v_{j H}^{1}\right)$ and $v_{j L}^{2}<f\left(v_{j L}^{1}\right)$. Then, apply Lemma 6 , $(\mathrm{T} 1 j)$, so that neither continuation value is on the frontier. Then, both $v_{j H}^{2}$ and $v_{j L}^{2}$ can be increased, again increasing $\bar{U}^{2}$ without affecting $\bar{U}^{1}$. The other case is analogous.

Lemma 8. Suppose that $(\mathbf{p}, \mathbf{q}, \mathbf{v})$ implements a Pareto efficient point in $\tilde{T}_{\text {On }}^{I}(V)$. For all $(j, k)$, if $v_{N}^{1}>v_{j k}^{1}>v_{E}^{1}$, then $p_{j k}=r$.

Proof. Suppose $p_{j k}<r$. Then we can increase $p_{j k}$ by $d \varepsilon$ and decrease $v_{j k}^{1}$ and $v_{j k}^{2}$ by $\frac{1}{\delta} d \varepsilon$ without affecting payoffs or on-schedule IC constraints. Then, we can improve utility by returning the continuation values to the frontier as in Lemma 7.

Proof of Proposition 6: Lemma 5 establishes that when the continuation value set has the shape $\left\{\left(u^{1}, u^{2}\right): u^{i} \square K\right\}$, the total cartel profits go down when firms use Pareto inefficient continuation values or prices. This logic can be applied directly here, observing that we are maximizing total profits because utility can be transferred across the firms (as in Proposition 3 ) under the conditions stated in the proposition.

Proof of Proposition 7: The proof is established by applying parts (ii) and (iii) of the following lemma. 
Lemma 9. Let $\mathbf{u}$ be a Pareto efficient point in $\tilde{T}^{I}(V)$, and let $(\mathbf{p}, \mathbf{q}, \mathbf{v})$ implement $\mathbf{u}$. (i) There exists an implementation of $\mathbf{u},(\hat{\mathbf{p}}, \hat{\mathbf{q}}, \hat{\mathbf{v}})$, such that: either (a) at least one off-schedule constraint binds, (b) IC-On1 $1_{D}$ binds, or else (c) $\hat{v}_{L L}^{2} \square \hat{v}_{H L}^{2}$ and $\hat{v}_{L H}^{2} \square \hat{v}_{H H}^{2}$; and, either (a), (d) IC-On2 binds, or (e) $\hat{v}_{L L}^{1} \square \hat{v}_{L H}^{1}$ and $\hat{v}_{H L}^{1} \square \hat{v}_{H H}^{1}$. (ii) If (p, q, v) satisfies pricing efficiency, or, more generally, $\Pi^{i}(H, H ; \mathbf{z}) \square \Pi^{i}(L, H ; \mathbf{z})$ for each $i$, and further continuation-value Pareto efficiency holds, then there exists an implementation of $\mathbf{u},(\hat{\mathbf{p}}, \hat{\mathbf{q}}, \hat{\mathbf{v}})$, such that $\hat{\mathbf{q}}=\mathbf{q}$ and either (a) at least one off-schedule constraint binds, or (b) IC-Oni $i_{D}$ binds for each firm, and unless $\bar{q}_{L}^{i}=\bar{q}_{H}^{i}$, ICOni $i_{U}$ is slack. (iii) If the assumptions of part (ii) hold, and if in addition, $\bar{q}_{L}^{i}>\bar{q}_{H}^{i}$ for each firm $i$, there exists an implementation of $\mathbf{u},(\hat{\mathbf{p}}, \hat{\mathbf{q}}, \hat{\mathbf{v}})$, such that $\hat{\mathbf{q}}=\mathbf{q}$ and either (a) at least one off-schedule constraint binds, or (b) IC-Oni $i_{D}$ binds for each firm $i$ and $\hat{v}_{L H}^{1} \square \hat{v}_{j j}^{1} \square \hat{v}_{H L}^{1}$ for $j \in\{L, H\}$.

Proof. Define

$$
L^{i}=\bar{q}_{L}^{i}-\bar{q}_{H}^{i} ; R_{B}^{i}=\delta\left(\bar{v}_{L}^{i}-\bar{v}_{H}^{i}\right) ; R_{A}^{1}=\sum_{k \in\{L, H\}} \eta_{k}\left(q_{L k}^{1} p_{L k}-q_{H k}^{1} p_{H k}\right)
$$

and likewise for $R_{A}^{2}$. Let $R^{i}=R_{A}^{i}+R_{B}^{i}$. Then IC-On $i_{D}$ can be written $L^{i} \theta_{H} \geq R^{i}$, while IC-On $i_{U}$ can be written $R^{i} \geq L^{i} \theta_{L}$. (i) Suppose that for $(\mathbf{p}, \mathbf{q}, \mathbf{v}), \mathrm{IC}-\mathrm{On} 1_{D}$ is slack and $v_{L k}^{2}>v_{H k}^{2}$ for some $k$. Then bringing together $v_{L k}^{2}$ and $v_{H k}^{2}$ according to trick (T2j) of Lemma 6 does not affect firm 2 . Given the convexity of the set of feasible continuation values, we can find corresponding feasible values of $\hat{v}_{L k}^{1}$ and $\hat{v}_{H k}^{1}$ whereby $R^{1}$ and $\vec{U}^{1}$ increase weakly; these do not violate on-schedule incentives for firm 1 since IC-On $1_{D}$ is slack. Thus, we can perform such adjustments until either (b) or (c) holds, or else (a) does, so that the off-schedule constraints prevent the adjustments. A similar logic holds for the case where IC-On $2_{D}$ is slack.

(ii) Lemma 3 implies $\bar{q}_{L}^{i} \geq \bar{q}_{H}^{i}$. If $\bar{q}_{L}^{i}=\bar{q}_{H}^{i}$, then simple calculations verify that IC-On $i_{D}$ and IC-On $i_{U}$ bind, while if $\bar{q}_{L}^{i}>\vec{q}_{H}^{i}$, only one on-schedule constraint can bind for each firm. Now suppose $\bar{q}_{L}^{i}>\vec{q}_{H}^{i}$. If prices are efficient, $L^{i} \theta_{H}-R_{A}^{i}=L^{i}\left(\theta_{H}-r\right)<0$, so that IC-On $i_{D}$ implies $R_{B}^{i}<0$. Since $\Pi^{i}(L, H ; \mathbf{z})-\Pi^{i}(H, H ; \mathbf{z})=L^{i} \theta_{H}-R_{A}^{i}$, the condition $\Pi^{i}(L, H ; \mathbf{z}) \square \Pi^{i}(H, H ; \mathbf{z})$ also implies $R_{B}^{i}<0$. Consider firm 1 . If $(\mathbf{p}, \mathbf{q}, \mathbf{v})$ is compatible with $R_{B}^{1}<0$, then we cannot have $v_{L L}^{1} \geq v_{H L}^{1}$ and $v_{L H}^{1} \geq v_{H H}^{1}$. Using continuation-value efficiency, the latter pair of inequalities are equivalent to those in part (i)(c). But, by part (i), there must then exist a $(\hat{\mathbf{p}}, \hat{\mathbf{q}}, \hat{\mathbf{v}})$ such that either (i)(a) or (i)(b) holds. The case of firm 2 is analogous. Finally, if IC-On $i_{D}$ binds and $\bar{q}_{L}^{i}>\bar{q}_{H}^{i}$ for each $i$, then each IC-On $i_{U}$ is slack.

(iii) Apply part (ii) to derive a scheme whereby either the downward on-schedule constraints bind and the upward on-schedule constraints are slack, or else the off-schedule constraints bind. Call this scheme $(\mathbf{p}, \mathbf{q}, \mathbf{v})$. Suppose that the off-schedule constraints are slack. Suppose further that $v_{j L}^{1}<v_{j H}^{1}$ (and thus, $v_{j L}^{2}>v_{j H}^{2}$ ). Following the logic of part (ii), we must have $v_{-j L}^{2}<v_{-j H}^{2}$ (and thus $v_{-j L}^{1}>v_{-j H}^{1}$ ), or else IC-On2 ${ }_{D}$ would be violated. Then, decreasing $v_{j H}^{1}$ by $\eta_{L}\left(v_{j H}^{1}-v_{j L}^{1}\right)$ and increasing $v_{j L}^{1}$ by $\eta_{H}\left(v_{j H}^{1}-v_{j L}^{1}\right)$ keeps $\bar{U}^{1}$ and firm 1's on-schedule constraints unaffected, while moving the continuation values for firm 2 along the frontier weakly increases $\bar{U}^{2}$ since $f$ is concave, and it relaxes $\mathrm{IC}-\mathrm{On} 2_{D}$ (though it may violate $\mathrm{IC}-\mathrm{On} 2_{U}$ ). Next, decrease $v_{-j L}^{1}$ at rate $\eta_{H}$ and increase $v_{-j H}^{1}$ at rate $\eta_{L}$, and move firm 2's continuation values along the frontier, until IC-On $2_{D}$ binds again (by the logic of part (ii), IC-On2 $2_{D}$ will bind with $\hat{v}_{-j L}^{2}<\hat{v}_{-j H}^{2}$ ). This leaves firm 1's on-schedule incentive constraints and $\bar{U}^{1}$ unchanged, while it weakly increases $\bar{U}^{2}$ since $f$ is concave. For this new scheme, either an off-schedule constraint binds, or else we have a feasible, weakly Pareto dominant scheme where the downward on-schedule constraints bind, 
and $\hat{v}_{j L}^{1}=\hat{v}_{j H}^{1}$ and $\hat{v}_{-j L}^{1}>\hat{v}_{-j H}^{1}$. Similar arguments establish that if $v_{L k}^{1}>v_{H k}^{1}$, we can find a weakly Pareto dominant scheme whereby the downward on-schedule constraints bind, and further $\hat{v}_{L k}^{2} \geq \hat{v}_{H k}^{2}$ for each $k$, and thus $\hat{v}_{L k}^{1} \square \hat{v}_{H k}^{1}$, establishing our result.

Proof of Proposition 8: First, suppose $q_{L H}^{1}<1$. Add $\frac{\delta}{p_{L H}-\theta_{L}} \varepsilon$ to $q_{L H}^{1}$ and subtract $\varepsilon$ from $v_{L H}^{1}$. If $v_{L H}$ is on the Pareto frontier and $\Delta_{\varepsilon}^{-} f\left(v_{L H}^{1}\right)>-1$, move $v_{L H}^{2}$ along the frontier of $V$. Otherwise, raise $v_{L H}^{2}$ by $\varepsilon$ (this is possible by convexity of the set of feasible continuation values, and since satisfaction of (4.2) implies $\left.v_{L H}^{1}>v_{N}^{1}\right)$. This does not affect $\bar{U}^{1}$. Consider now the effect on the interim expected utility of both firms: $U^{1}(H, H ; \mathbf{z}), U^{1}(H, L ; \mathbf{z})$, and $U^{1}(L, L ; \mathbf{z})$ are unchanged; and $U^{1}(L, H ; \mathbf{z})$ decreases. $U^{2}(L, H ; \mathbf{z})$ and $U^{2}(L, L ; \mathbf{z})$ are unchanged. $U^{2}(H, H ; \mathbf{z})$ goes up if $-\left(p_{L H}-\theta_{H}\right) /\left(p_{L H}-\theta_{L}\right)-\max \left(-1, \Delta_{\varepsilon}^{-} f\left(v_{L H}^{1}\right)\right)>0$, which when rearranged gives (4.2). $U^{2}(H, L ; \mathbf{z})$ goes down if $v_{L H}^{2}$ increases by no more than $\varepsilon$, which it does by construction. Thus, all of the on-schedule incentive-compatibility constraints are relaxed. Finally, none of firm 1's off-schedule constraints are affected by this shift, and firm 2's off-schedule constraints are relaxed. To see the result for $q_{H L}^{1}$, we perform an analogous trick, subtracting $\frac{\delta}{p_{H L}-\theta_{H}} d \varepsilon$ from $q_{H L}^{1}$ and adding $d \varepsilon$ to $v_{H L}^{1}$, and noting that satisfaction of (4.3) implies that $v_{H L}^{1}<v_{E}^{1}$ (recalling that in the definition of $f$, we specified a large negative slope for $f$ when $v_{j k}^{1} \geq v_{E}^{1}$ ).

Proof of Proposition 9: Under the assumptions of the proposition, utility is fully transferable across the firms, and we can simply maximize the sum of firm utilities. Doing so leads to a symmetric scheme across states $(L, H)$ and $(H, L)$, with one firm being favored over another in states $(H, H)$ and $(L, L)$, if at all. Now imagine lowering $q_{L H}^{1}$ and raising $q_{H L}^{1}$ by $\varepsilon$, and then adjusting $v_{L H}^{1}$ and $v_{H L}^{2}$ upward by $\zeta$ until both firms' downward IC constraints bind again. The opponents' continuation values are moved along the frontier. Solving for $\zeta$, we obtain:

$$
\frac{1}{1-\eta_{L}\left(1-\Delta_{\varepsilon}^{+} f\left(v_{L H}^{1}\right)\right)} \frac{\varepsilon\left(p_{L H}-\theta_{H}\right)}{\delta} .
$$

It can be shown using algebraic manipulation that the first inequality in the statement of the proposition is necessary and sufficient for this change to lower total firm profit. The second inequality is necessary and sufficient for the firms' joint profit to decrease if we reverse this change.

Proof of Proposition 10: The linear self-generating set of equilibria constructed in the proof of Proposition 1 implements the endpoints of the segment, $(x, y)$ and $(y, x)$, using schemes that have market shares $q_{L H}^{1}=1, q_{j k}^{1}=0$ for all other $(j, k)$. Consider a scheme whereby firm 1 chooses $\rho^{1}\left(\theta_{H}\right)=r$ and $\rho^{1}\left(\theta_{L}\right)=r-2 \varepsilon$, and $\rho^{2}\left(\theta_{H}\right)=r-\varepsilon$ and $\rho^{2}\left(\theta_{L}\right)=r-3 \varepsilon$. Using this scheme, the market shares are assigned appropriately. Further, each firm's announced price differs by state, so that continuation values can be contingent purely on prices. Thus, communication is not required to implement the scheme. Since firms can draw from a convex set of continuation values, all continuation values in between $(x, y)$ and $(y, x)$ are available to the firms, and the linear set is self-generating.

Proof of Proposition 11: This follows from Lemma 5, since the set of Symmetric PPE values is contained in the set $V^{\prime}=\left\{\left(u^{1}, u^{2}\right):\left(u^{1}, u^{2}\right) \square \sup \left\{u \in V^{S}\right\}\right\}$, and Lemma 5 indicates that the highest point in $T^{S}\left(V^{\prime}\right)$ is implemented as described in (i) and (ii). Since the scheme described in (i) is still feasible when we take $V^{S}$ as the set of feasible continuation values, it must be optimal. The rigid-price scheme clearly requires no communication; the scheme in (ii) can be implemented without communication, using $\rho^{i}\left(\theta_{H}\right)=r$ and $\rho^{i}\left(\theta_{L}\right)=\hat{p}$.

Proof of Proposition 12: (i) Under the stated conditions, the frontier has an open interval with slope -1 , and thus it is more efficient to use continuation values rather than inefficient bribes. 
(ii) Proposition 1 establishes a critical discount factor beyond which first-best can be attained without bribes; thus, if bribes are at all inefficient, they are not used even if they are available.

Proof of Proposition 13: This follows as in Proposition 8.

Proof of Proposition 14: (i) This follows as in Lemma 5 and Proposition 8; the availability of bribes does not change the tradeoff between productive inefficiency and inefficient continuation values. (ii) When all of the off-schedule constraints are cleared, productive efficiency can be implemented using bribes. The optimality of bribes for $\gamma$ in the specified region follows exactly as in Proposition 8, replacing continuation values with bribes. Consider a scheme that uses equal market-sharing and no bribes in the event of ties and productive efficiency, and $b_{L H}^{1}=$ $\left(r-\theta_{H}\right) /\left(2+(1-\gamma) \eta_{H}\right)$. It can be verified that this scheme satisfies all of the on-schedule IC constraints. The critical discount factor can be computed simply by checking the off-schedule constraints.

\section{References}

Abreu, Dilip, David Pearce and Ennio Stacchetti, "Optimal Cartel Equilibria with Imperfect Monitoring", Journal of Economic Theory, 39.1, June 1986, pp. 251-69.

Abreu, Dilip, David Pearce and Ennio Stacchetti, "Toward a Theory of Discounted Repeated Games with Imperfect Monitoring," Econometrica, 58.5, September 1990, pp. 1041-63.

Aoyagi, Masaki, "Bid Rotation in a Model of Repeated Auctions," Mimeo, 1998, University of Pittsburgh.

Athey, Susan, Kyle Bagwell, and Chris Sanchirico, 1998, "Collusion and Price Rigidity," MIT Working Paper no. 98-23.

Atkeson, Andrew and Robert E. Lucas, "On Efficient Distribution with Private Information," Review of Economic Studies, 59.3, July 1992, pp. 427-53.

Bernheim, B. Douglas and Michael Whinston, "Multimarket Contact and Collusive Behavior," RAND Journal of Economics, 21, 1990, 1-26.

Bork, Robert H., "The Rule of Reason and The Per Se Concept: Price Fixing and Market Division," Yale Law Journal, 74.5, April 1965, 775-847.

Bork, Robert H., "The Rule of Reason and The Per Se Concept: Price Fixing and Market Division," Yale Law Journal, 75.3, January 1966, 373-475.

Business Week, "Justice Cartel Crackdown," July 27, 1998, pp. 50-51.

Cole, Harold L., and Narayana Kocherlakota, "Dynamic Games with Hidden Actions and Hidden States," Federal Reserve Bank of Minneapolis Research Department Staff Report 254, 1998.

Comanor, W.S. and M.A. Schankerman, "Identical Bids and Cartel Behavior," Bell Journal of Economics, 7, 1976, 281-6.

Compte, Olivier, "Communication in Repeated Games with Imperfect Private Monitoring," Econometrica, 66.3, May 1998, 597-626.

Cramton, Peter C. and Thomas R. Palfrey, "Cartel Enforcement with Uncertainty about Costs." International Economic Review, 31 (1) February 1990, pp. 17-47.

Damania, Richard and Bill Z. Yang, "Price Rigidity and Asymmetric Price Adjustment in a Repeated Oligopoly," Journal of Institutional and Theoretical Economics, 154, 1998, pp. 659-679. 
Doyle, Maura, and Christopher Snyder, "Information Sharing and Competition in the Motor Vehicle Industry," Mimeo, George Washington University, 1998.

d'Aspremont, Claude and L.A. Gerard-Varet, "Incentives and Incomplete Information," Journal of Public Economics, 11, 1979, 25-45.

Fudenberg, Drew, David J. Levine and Eric Maskin, "The Folk Theorem with Imperfect Public Information," Econometrica, 62.5 September 1994, pp. 997-1039.

Genesove, David and Wallace Mullin, "Narrative Evidence on the Dynamics of Collusion: The Sugar Institute Case," Mimeo, 1998, Michigan State University.

Genesove

Minnesota Studies in Macroeconomics series, vol. 1 Minneapolis: University of Minnesota Press 1987, pp. 3-25.

Green, Edward J. and Robert H. Porter, "Noncooperative Collusion under Imperfect Price Information," Econometrica; 52.1 January 1984, pp. 87-100.

Holmstrom, Bengt and David Kreps, "Promises," mimeo, MIT, 1996.

Judd, Kenneth, and James Conklin, "Computing Supergame Equilibria," Mimeo, Hoover Institution, Stanford University, 1995.

Kandori, Michihiro and Hitoshi Matshushima, "Private Observation, Communication and Collusion," Econometrica, 66.3, May 1998, 627-52.

Kihlstrom, Richard and Xavier Vives, "Collusion by Asymmetrically Informed Firms," Journal of Economics and Management Strategy, 1 (2) 1992, pp. 371-96.

Kuhn, Kai-Uwe and Xavier Vives, "Information Exchanges Among Firms and Their Impact on Competition," mimeo, Barcelona: Institut d'Analisi Economica, June 1994.

Levinsohn, James, "Competition Policy and International Trade," in Fair Trade and Harmonization, J. Bhagwati and R. Hudec, eds., MIT Press, pp. 329-356, 1995.

McAfee, Preston and John McMillan, "Bidding Rings," American Economic Review; 82.3, June 1992, pp. 579-599.

McMillan, John , "Dango: Japan's Price-Fixing Conspiracies," Economics and Politics, November 1991, (3): 201-218.

McCutcheon, Barbara, "Do Meetings in Smoke-Filled Rooms Facilitate Collusion?" Journal of Political Economy 105 (2) April 1997, pp. 330-50.

Phelan, Christopher, and Robert M. Townsend, "Computing Multi-period, InformationConstrained Optima." Review of Economic Studies 58 (5), 1991, pp. 853-81.

Radner, Roy, "Monitoring Cooperative Agreements in a Repeated Principal-Agent Relationship," Econometrica, 49, 1981, pp. 1127-1148.

Roberts, Kevin, "Cartel Behavior and Adverse Selection," Journal of Industrial Economics, 33, 1985, pp. 401-413.

Rotemberg, Julio and Garth Saloner, "A Supergame-Theoretic Model of Price Wars During Booms," American Economic Review, 76, 1986, pp. 390-407. 
Shapiro, Carl, "Exchanges of Cost Information in Oligopoly," Review of Economic Studies, 53, 1986, pp. 433-446.

Sproul, Michael, "Antitrust and Prices," Journal of Political Economy, 101.4, 1993, pp. 741-754.

Stocking, George W. and Myron W. Watkins, Cartels in Action. Case Studies in International Business Diplomacy, New York: The Twentieth Century Fund, 1946.

Vives, Xavier, "Duopoly Information Equilibrium: Cournot and Bertrand," Journal of Economic Theory, 34, 1984, 71-94.

Wang, Cheng, "Dynamic Insurance with Private Information and Balanced Budgets," $R e$ view of Economic Studies ; 62.4, October 1995, pp. 577-95. 



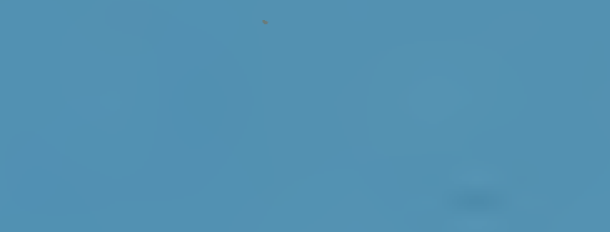

a

ad 110 L. 




DEC 2009 Date Due


